\title{
Tangent cones and local geometry of the representation and character varieties of knot groups
}

\author{
LEILA BEN ABDELGHANI
}

\begin{abstract}
Let $K$ be a knot in the 3-sphere $S^{3}$. We calculate explicitly the tangent cone to the representation variety at an abelian representation which corresponds to a double root of the Alexander polynomial. We also describe the local structure of the representation and character varieties.
\end{abstract}

$57 \mathrm{M} 25,57 \mathrm{M} 27$

\section{Introduction}

Given a knot $K$ in $S^{3}$, we let $M=S^{3} \backslash K$ denote its complement and $\Gamma:=\pi_{1}(M)$ denote the fundamental group of $M$.

Gordon and Luecke [13] showed that knots are determined by their complements and Whitten [24] proved that the classification of prime knots reduces to that of their knot groups. So one way to the understanding of knots is the study of their groups.

Research has shown that many topological properties of a knot are encoded in the representation and character varieties of its group, so it is of interest to study their basic properties.

We denote by $R(\Gamma):=\operatorname{Hom}\left(\Gamma, \mathrm{SL}_{2}(\mathbb{C})\right.$ ) the representation variety of $\Gamma$ (see Section 2.1) and by $S(\Gamma)$ the set of abelian representations, ie those which factor through $\Phi: \Gamma \rightarrow \Gamma / \Gamma^{\prime}=T$, where $T$ is an infinite cyclic group. Note that $S(\Gamma) \subset R(\Gamma)$ is an irreducible algebraic component of $R(\Gamma)$ (see Klassen [18]).

A representation $\rho: \Gamma \rightarrow \mathrm{SL}_{2}(\mathbb{C})$ is called irreducible if the only subspaces of $\mathbb{C}^{2}$ which are invariant under $\rho(\Gamma)$ are $\{0\}$ and $\mathbb{C}^{2}$. We denote $R^{\text {irr }}(\Gamma) \subset R(\Gamma)$ the subset of irreducible representations and $R^{\text {red }}(\Gamma)$ the subset of reducible ones. Note that $R^{\mathrm{irr}}(\Gamma)$ is Zariski open and $R^{\mathrm{red}}(\Gamma)$ is Zariski closed in $R(\Gamma)$ (see Culler and Shalen [11]).

The aim of this article is to study the local structure of the representation space of $\Gamma$ in the Lie group $\mathrm{SL}_{2}(\mathbb{C})$ in the neighborhood of an abelian representation. 
By a result of Thurston, under certain conditions, one can deform an irreducible representation nontrivially but there is no general theorem which allows the deformation of reducible representations. Many authors have studied this problem, for example, the author [2; 3], the author and Lines [4], Frohman and Klassen [12], Herald [14], Heusener and Kroll [15], Heusener, Porti and Suárez Peiró [17] and Shors [22]. The latter (in an unpublished work) has considered the case when the Alexander module has cyclic torsion. The present paper is also concerned with this case.

A first approximation to the local structure of the representation variety at a given representation is given by its Zariski tangent space and a much finer invariant is the tangent cone. In this article, we calculate explicitly the tangent cone to the representation variety at an abelian representation which corresponds to a root $\alpha$ of the Alexander polynomial $\Delta_{1}$ of the knot $K$ and such that the $(t-\alpha)$-torsion of the Alexander module $H_{1}(\widetilde{M}, \mathbb{C})$ is cyclic of order 2 . Moreover, we give an approximation to the tangent cone in the case of cyclic $(t-\alpha)$-torsion of the Alexander module of order $r, r \geq 3$.

More precisely, let $\alpha=\lambda^{2}$ be a root of $\Delta_{1}$ and suppose that the $(t-\alpha)$-torsion of the Alexander module is of the form

$$
\frac{\mathbb{C}\left[t, t^{-1}\right]}{(t-\alpha)^{r}} \oplus \frac{\mathbb{C}\left[t, t^{-1}\right]}{\left(t-\alpha^{-1}\right)^{r}}, \quad r \geq 2 .
$$

Let $\rho_{\lambda}: \Gamma \rightarrow \mathrm{SL}_{2}(\mathbb{C})$ the abelian representation given by $\rho_{\lambda}(\mu)=\operatorname{diag}\left(\lambda, \lambda^{-1}\right) \in$ $\mathrm{SL}_{2}(\mathbb{C})$.

As an approximation to the tangent cone $\mathrm{TC}_{\rho_{\lambda}}$, we have a descending sequence of cones included in the Zariski tangent space $Z^{1}\left(\Gamma, \mathfrak{s l}_{\rho_{\lambda}}\right)$. The first of this cones is the quadratic cone $\mathrm{QC}_{\rho_{\lambda}}$ and the second one is the cubic cone $\mathrm{TC}_{\rho_{\lambda}}^{3}$.

We shall prove the following theorem:

Theorem 1.1 Assume that the $(t-\alpha)$-torsion is cyclic of order $r \geq 2$.

(1) If $r=2$ then $\mathrm{TC}_{\rho_{\lambda}}=\mathrm{TC}_{\rho_{\lambda}}^{3} \varsubsetneqq \mathrm{QC}_{\rho_{\lambda}}=Z^{1}\left(\Gamma, \mathfrak{s l}_{\rho_{\lambda}}\right)$.

(2) If $r \geq 3$ then $\mathrm{TC}_{\rho_{\lambda}} \varsubsetneqq \mathrm{TC}_{\rho_{\lambda}}^{3}=\mathrm{QC}_{\rho_{\lambda}}=Z^{1}\left(\Gamma, \mathfrak{s l}_{\rho_{\lambda}}\right)$.

Let $\phi_{\lambda}: \Gamma \rightarrow \mathrm{SL}_{2}(\mathbb{C})$ be the reducible nonabelian representation with the same character as $\rho_{\lambda}$ and given by $\phi_{\lambda}=\exp \left(V_{+}\right) \rho_{\lambda}$ (see Section 4.2). Note that $\phi_{\lambda}$ is unique up to conjugation. The study of the local structure of the representation variety near the abelian representation $\rho_{\lambda}$ and near the reducible nonabelian representation $\phi_{\lambda}$ goes simultaneously. 
Proposition 1.2 Suppose that $r=2$; then the representation $\phi_{\lambda}$ is scheme reduced. Moreover:

(1) If $\operatorname{dim}_{\mathbb{C}} Z^{1}\left(\Gamma, \mathfrak{s l}_{\phi_{\lambda}}\right)=4$ then $\phi_{\lambda}$ is a smooth point of $R(\Gamma)$ which is contained in a 4-dimensional component $R_{\lambda}(\Gamma)$; so

$$
\mathrm{TC}_{\phi_{\lambda}}=\mathrm{QC}_{\phi_{\lambda}}=T_{\phi_{\lambda}}^{\mathrm{Zar}} R(\Gamma)=Z^{1}\left(\Gamma, \mathfrak{s l}_{\phi_{\lambda}}\right) .
$$

Further, in this case, the abelian representation $\rho_{\lambda}$ is contained in exactly two irreducible components of $R(\Gamma)$, namely $S(\Gamma)$ and $R_{\lambda}(\Gamma)$, and $\rho_{\lambda}$ is a smooth point of both varieties.

(2) If $\operatorname{dim}_{\mathbb{C}} Z^{1}\left(\Gamma, \mathfrak{s l}_{\phi_{\lambda}}\right)=5$, then $\phi_{\lambda}$ is a singular point of $R(\Gamma)$ which is contained in, at least, two 4-dimensional components of $\overline{R^{\operatorname{irr}}(\Gamma)}$ and

$$
\mathrm{TC}_{\phi_{\lambda}}=\mathrm{QC}_{\phi_{\lambda}} \varsubsetneqq T_{\phi_{\lambda}}^{\mathrm{Zar}} R(\Gamma)=Z^{1}\left(\Gamma, \mathfrak{s l}_{\phi_{\lambda}}\right) .
$$

Further, in this case, the abelian representation $\rho_{\lambda}$ is contained in at least 3 components of $R(\Gamma)$.

The variety of characters $X(\Gamma)$ of a finitely generated group $\Gamma$ is the quotient in the algebraic category of the action of $\mathrm{SL}_{2}(\mathbb{C})$ by conjugation on the variety of representations $R(\Gamma)$. We denote the projection by $\pi: R(\Gamma) \rightarrow X(\Gamma)$. For a representation $\rho \in R(\Gamma)$, its projection onto $X(\Gamma)$, denoted by $\chi_{\rho}$, is called the character of $\rho$ and may be interpreted as a map $\chi_{\rho}: \Gamma \rightarrow \mathbb{C} ; \gamma \mapsto \operatorname{tr}(\rho(\gamma))[11]$.

We denote by $Y(\Gamma)$ the projection of $S(\Gamma)$ onto the character variety $X(\Gamma)$ and by $X^{\mathrm{irr}}(\Gamma):=\pi\left(R^{\mathrm{irr}}(\Gamma)\right)$ the set of irreducible characters of $\Gamma$. Note that $X^{\mathrm{irr}}(\Gamma)$ is Zariski open in $X(\Gamma)$ [11]. We also use the notation $\chi_{\lambda}:=\chi_{\phi_{\lambda}}=\chi_{\rho_{\lambda}}$ and $X_{\lambda}(\Gamma):=$ $\pi\left(R_{\lambda}(\Gamma)\right)$.

As an application to the explicit description of the tangent cone, we give a description of the local geometry of the representation and character varieties in the case of a cyclic $(t-\alpha)$-torsion of the Alexander module of order 2.

Theorem 1.3 Assume that the $(t-\alpha)$-torsion is cyclic of order 2 .

(1) Suppose that $\operatorname{dim}_{\mathbb{C}} Z^{1}\left(\Gamma, \mathfrak{s l}_{\phi_{\lambda}}\right)=4$. Then the curves $X_{\lambda}(\Gamma)$ and $Y(\Gamma)$ are the unique irreducible components of $X(\Gamma)$ that contain $\chi_{\lambda}$. In addition, $\chi_{\lambda}$ is a smooth point of both curves, the two curves are tangent at $\chi_{\lambda}$ and $T_{\chi_{\lambda}}^{\mathrm{Zar}} \overline{X^{\mathrm{irr}}(\Gamma)} \cong$ $H^{1}\left(\Gamma, \mathfrak{s l}_{\phi_{\lambda}}\right)$.

(2) Suppose that $\operatorname{dim}_{\mathbb{C}} Z^{1}\left(\Gamma, \mathfrak{s l}_{\phi_{\lambda}}\right)=5$. Then $\chi_{\lambda}$ is contained in at least 2 irreducible components of $\overline{X^{\text {irr }}(\Gamma)}$ and is not a smooth point of the character variety of irreducible representations. 
The paper is organised as follows. In Section 2 the basic notation and facts are introduced. In Section 3, we recall some results about abelian representations. The proof of the Theorem 1.1 is presented in Section 4. Section 5 includes the proofs of Proposition 1.2 and Theorem 1.3 and contains examples. The last section, Section 6, is devoted to the proof of a technical result.

Acknowledgments I would like to thank an anonymous referee for his careful and thorough reading of the manuscript and for providing useful suggestions for improving the paper. I am also grateful to Daniel Lines, Michael Heusener and Houda Lahouar for their friendly support and their useful comments concerning a first version of this paper.

\section{Representation spaces and deformations}

In this section, we recall some notation and facts concerning the representation and character varieties and the formal deformations. We introduce also the notion of $i$-th tangent cone at a representation as well as the terminology of quadratic and strongly nonquadratic singularity of the representation variety.

\subsection{Representation spaces}

Given a finitely presented group $\Gamma=\left\langle S_{1}, \ldots, S_{n} \mid R_{1}, \ldots, R_{m}\right\rangle$ and a complex Lie group $G$, the space of all representations (ie homomorphisms) of the group $\Gamma$ into $G$ is denoted by $R(\Gamma, G)$. The space $R(\Gamma, G)$ is equipped with the compact open topology and can be identified with the following subspace of $G^{n}$ :

$$
R(\Gamma, G):=\left\{\left(g_{1}, \ldots, g_{n}\right) \mid R_{j}\left(g_{1}, \ldots, g_{n}\right)=E, j=1, \ldots, m\right\},
$$

where $E$ is the unit in $G$. The relations $R_{j}$ give a smooth map $R: G^{n} \rightarrow G^{m}$ and $R(\Gamma, G)$ can be identified with $R^{-1}(E, \ldots, E)$.

The space $R(\Gamma, G)$ inherits an algebraic structure if $G$ is an algebraic group. It is easy to see that the map $R: G^{n} \rightarrow G^{m}$ is polynomial in the ambient coordinates. The set $R^{-1}(E, \ldots, E)$ is therefore an affine algebraic set and the induced algebraic structure on $R(\Gamma, G)$ does not depend on the presentation of $\Gamma[19]$. The space $R(\Gamma, G)$ carries two topologies, the Zariski and the complex or classical topology.

Let $\rho: \Gamma \rightarrow G$ be a representation. The Lie algebra $\mathcal{G}$ of $G$ is turned via Ad $\circ \rho$ into a $\Gamma$-module denoted by $\mathcal{G}_{\rho}$, ie for all $X \in \mathcal{G}$ and for $\gamma \in \Gamma$, we have $\gamma \cdot X=$ $\rho(\gamma) X \rho(\gamma)^{-1}$. We denote by $C^{n}\left(\Gamma, \mathcal{G}_{\rho}\right):=\left\{u: \Gamma^{n} \rightarrow \mathcal{G}\right\}$ the space of $n$-cochains and the coboundary operator is denoted by $\partial: C^{n}\left(\Gamma, \mathcal{G}_{\rho}\right) \rightarrow C^{n+1}\left(\Gamma, \mathcal{G}_{\rho}\right)$. Let $B^{*}\left(\Gamma, \mathcal{G}_{\rho}\right)$ 
(resp. $Z^{*}\left(\Gamma, \mathcal{G}_{\rho}\right)$, resp. $\left.H^{*}\left(\Gamma, \mathcal{G}_{\rho}\right)\right)$ be the coboundaries (resp. cocycles, resp. cohomology groups) of $\Gamma$ with coefficients in $\mathcal{G}_{\rho}$.

We denote by $T_{\rho}^{\mathrm{Zar}} R(\Gamma, G)$ the Zariski tangent space of the variety $R(\Gamma, G)$ at the representation $\rho$. It was observed by Weil that there is a natural inclusion $T_{\rho}^{\mathrm{Zar}} R(\Gamma, G) \hookrightarrow$ $Z^{1}\left(\Gamma, \mathcal{G}_{\rho}\right)$ : for each analytic path $\rho_{t}: \Gamma \rightarrow G$ such that $\rho_{0}=\rho$, the map $u: \Gamma \rightarrow \mathcal{G}$ given by

$$
u(\gamma):=\left.\frac{d\left(\rho_{t}(\gamma)\right)}{d t}\right|_{t=0} \rho^{-1}(\gamma)
$$

is a cocycle [23]. It is also proved in [19] that the space $Z^{1}\left(\Gamma, \mathcal{G}_{\rho}\right)$ can be identified with the tangent space of the scheme of all representations of $\Gamma$ to $G$ at $\rho$. In general, the space of cocycles is larger then the Zariski tangent space. A representation $\rho: \Gamma \rightarrow G$ is called scheme reduced if the inclusion $T_{\rho}^{\mathrm{Zar}} R(\Gamma, G) \hookrightarrow Z^{1}\left(\Gamma, \mathcal{G}_{\rho}\right)$ is an isomorphism. Even for a scheme reduced representation, the space $Z^{1}\left(\Gamma, \mathcal{G}_{\rho}\right)$ gives only a first approximation to the local structure of the representation variety. A much finer invariant is the tangent cone $\mathrm{TC}_{\rho} R(\Gamma, G)$, that is the set of all cocycles $u \in Z^{1}\left(\Gamma, \mathcal{G}_{\rho}\right)$ which are tangent to an analytic path in $R(\Gamma, G)$.

Definition 2.1 A representation $\rho: \Gamma \rightarrow G$ is called regular if the tangent cone is equal to $Z^{1}\left(\Gamma, \mathcal{G}_{\rho}\right)$. Otherwise, $\rho$ is called singular.

We are interested in the case where $\Gamma$ is a knot group and $G$ is the Lie group $\mathrm{SL}_{2}(\mathbb{C})$ which we denote SL for simplicity.

\subsection{Formal deformations and cones}

Let $M_{1}$ and $M_{2}$ be two $\Gamma$-modules. The cup product of two cochains $u \in C^{p}\left(\Gamma, M_{1}\right)$ and $v \in C^{q}\left(\Gamma, M_{2}\right)$ is the cochain $u \smile v \in C^{p+q}\left(\Gamma, M_{1} \otimes M_{2}\right)$ defined by

$$
u \smile v\left(\gamma_{1}, \ldots, \gamma_{p+q}\right):=u\left(\gamma_{1}, \ldots, \gamma_{p}\right) \otimes \gamma_{1} \cdots \gamma_{p} \cdot v\left(\gamma_{p+1}, \ldots, \gamma_{p+q}\right) .
$$

It is possible to combine the cup product with any $\mathbb{C}[\Gamma]-$ bilinear map $b: M_{1} \otimes M_{2} \rightarrow$ $M_{3}$. So we obtain a cup product $C^{p}\left(\Gamma, M_{1}\right) \otimes C^{q}\left(\Gamma, M_{2}\right) \rightarrow C^{p+q}\left(\Gamma, M_{3}\right)$ [8].

We are mainly interested in the case where the $\Gamma$-module $\mathcal{G}=M_{1}=M_{2}$ is also a Lie algebra. Then by using the Lie brackets of $\mathcal{G}$ as a bilinear map we obtain the cup bracket of two cochains which will be denoted by $u \smile v$. Note that the cup bracket is not associative on the cochain level.

Definition 2.2 A formal deformation of $\rho: \Gamma \rightarrow G$ is a representation $\rho_{t}: \Gamma \rightarrow$ $G(\mathbb{C} \llbracket t \rrbracket)$ such that $\rho_{0}=\rho$. Here $\mathbb{C} \llbracket t \rrbracket$ denotes the ring of formal power series and $\rho_{0}: \Gamma \rightarrow G$ is the evaluation of $\rho_{t}$ at $t=0$. 
Every formal deformation $\rho_{t}$ of $\rho: \Gamma \rightarrow G$ can be written in the form $\rho_{t}(\gamma)=$ $\exp \left(t v_{1}(\gamma)+t^{2} v_{2}(\gamma)+\cdots\right) \rho(\gamma)$ where $v_{i}: \Gamma \rightarrow \mathcal{G}$ are maps, ie elements of $C^{1}\left(\Gamma, \mathcal{G}_{\rho}\right)$ and Weil's construction gives that $v_{1} \in Z^{1}\left(\Gamma, \mathcal{G}_{\rho}\right)$ is a cocycle.

More generally, we have the following:

Lemma 2.3 Let $\rho: \Gamma \rightarrow G$ be a representation. Then $\rho_{t}: \Gamma \rightarrow G(\mathbb{C} \llbracket t \rrbracket)$ given by $\rho_{t}=\exp \left(\sum_{i \geq 1} t^{i} v_{i}\right) \rho$ is a homomorphism if and only if

$$
\left\{\begin{array}{l}
-\partial v_{1}=0 \\
-\partial v_{n}=\frac{1}{2} \sum_{k=1}^{n-1} v_{k} \smile v_{n-k}+\sum_{j=3}^{n} A_{j}\left(v_{1}, \ldots, v_{n-2}\right), \text { for } n \geq 2,
\end{array}\right.
$$

where, for $\gamma_{1}, \gamma_{2} \in \Gamma, A_{j}\left(v_{1}, \ldots, v_{n-2}\right)\left(\gamma_{1}, \gamma_{2}\right)$ is a sum of monomials which are multiple brackets of exactly $j$-terms $v_{i}\left(\gamma_{1}\right)$ or $\gamma_{1} \cdot v_{l}\left(\gamma_{2}\right), 1 \leq i, l \leq n-2$ [2].

For all $n \geq 2$, denote by $\zeta_{n}\left(v_{1}, \ldots, v_{n-1}\right)$ the 2 -cochain on $\Gamma$ such that

$$
\zeta_{n}\left(v_{1}, \ldots, v_{n-1}\right)=\frac{1}{2} \sum_{k=1}^{n-1} v_{k} \smile v_{n-k}+\sum_{j=3}^{n} A_{j}\left(v_{1}, \ldots, v_{n-2}\right) .
$$

It is easy to see that $\zeta_{n}\left(v_{1}, \ldots, v_{n-1}\right) \in Z^{2}\left(\Gamma, \mathcal{G}_{\rho}\right)$ (see [4, proof of Proposition 4.10]). Note that to construct a formal deformation of $\rho$ it suffices to solve the infinite sequence of obstructions

$$
\left\{\begin{array}{l}
-\partial v_{1}=0 \\
-\partial v_{n}=\zeta_{n}\left(v_{1}, \ldots, v_{n-1}\right), n \geq 2
\end{array}\right.
$$

The first of these obstructions is the cup product $\left\{v_{1} \smile v_{1}\right\}$ and each obstruction is defined in terms of the preceding solution on the cochain level and each taking values in $H^{2}\left(\Gamma, \mathcal{G}_{\rho}\right)$ [20].

Definition 2.4 We call a cocycle $v_{1} \in Z^{1}\left(\Gamma, \mathcal{G}_{\rho}\right)$ formally integrable if there exists a formal deformation $\rho_{t}$ with first order term $v_{1}$.

By a theorem of Artin [1], the tangent cone to $R(\Gamma, G)$ at $\rho$,

$\operatorname{TC}_{\rho} R(\Gamma, G)=\left\{\left.\frac{d \rho_{t} \rho^{-1}}{d t}\right|_{t=0} \mid \rho_{t}\right.$ is an analytic curve in $R(\Gamma, G)$ with endpoint $\left.\rho\right\}$ is exactly the set of formally integrable cocycles.

As an approximation to the tangent cone, we have a descending sequence of cones and the first of them is the quadratic cone. 
Definition 2.5 Let $\rho: \Gamma \rightarrow G$ be a representation. The quadratic cone at $\rho$ to $R(\Gamma, G)$ is the subset of $Z^{1}\left(\Gamma, \mathcal{G}_{\rho}\right)$ given by

$$
\mathrm{QC}_{\rho} R(\Gamma, G)=\left\{u \in Z^{1}\left(\Gamma, \mathcal{G}_{\rho}\right) \mid\{u \smile u\}=0 \in H^{2}\left(\Gamma, \mathcal{G}_{\rho}\right)\right\} .
$$

We say $R(\Gamma, G)$ has a quadratic singularity at $\rho$ if $\rho$ is singular and $\operatorname{TC}_{\rho} R(\Gamma, G)=$ $\mathrm{QC}_{\rho} R(\Gamma, G)$, ie the obstructions are at most quadratic.

Now let $u_{1} \in Z^{1}\left(\Gamma, \mathcal{G}_{\rho}\right)$ be a cocycle in the quadratic cone, ie $\left\{u_{1} \smile u_{1}\right\}=0$ in $H^{2}\left(\Gamma, \mathcal{G}_{\rho}\right)$. Then there exists a cochain $u_{2}: \Gamma \rightarrow \mathcal{G}_{\rho}$ such that $-\partial u_{2}=\frac{1}{2} u_{1} \smile u_{1}$. The cochain $u_{2}$ is only unique up to addition of a cocycle $Z \in Z^{1}\left(\Gamma, \mathcal{G}_{\rho}\right)$.

The third obstruction is given by the cocycle

$$
\begin{aligned}
\zeta_{3}\left(u_{1}, u_{2}\right)\left(\gamma_{1}, \gamma_{2}\right)=\frac{1}{2} u_{1} \smile u_{2}\left(\gamma_{1}, \gamma_{2}\right) & +\frac{1}{2} u_{2} \smile u_{1}\left(\gamma_{1}, \gamma_{2}\right) \\
& +\frac{1}{12}\left[\left[u_{1}\left(\gamma_{1}\right), \gamma_{1} \cdot u_{1}\left(\gamma_{2}\right)\right], \gamma_{1} \cdot u_{1}\left(\gamma_{2}\right)-u_{1}\left(\gamma_{1}\right)\right] .
\end{aligned}
$$

Its existence depends on that of $u_{2}$ and is unique up to adding elements of the form $\frac{1}{2} Z \smile u_{1}+\frac{1}{2} u_{1} \smile Z$ where $Z \in Z^{1}\left(\Gamma, \mathcal{G}_{\rho}\right)$.

The cubic cone at $\rho$ to $R(\Gamma, G)$ is by definition the set

$$
\begin{aligned}
& \operatorname{TC}_{\rho}^{3} R(\Gamma, G):=\left\{u_{1} \in \mathrm{QC}_{\rho}(\Gamma) \mid \exists u_{2} \in C^{1}\left(\Gamma, \mathcal{G}_{\rho}\right)\right. \text { such that } \\
& \left.\zeta_{3}\left(u_{1}, u_{2}\right) \text { exists and }\left\{\zeta_{3}\left(u_{1}, u_{2}\right)\right\}=0 \in H^{2}\left(\Gamma, \mathcal{G}_{\rho}\right)\right\} .
\end{aligned}
$$

More generally, for $i \geq 3$, we define the $i$-th tangent cone at $\rho$ to $R(\Gamma, G)$ as

$$
\begin{aligned}
\operatorname{TC}_{\rho}^{i} R(\Gamma, G):= & \left\{u_{1} \in Z^{1}\left(\Gamma, \mathcal{G}_{\rho}\right) \mid \exists u_{2}, \ldots, u_{i-1} \in C^{1}\left(\Gamma, \mathcal{G}_{\rho}\right)\right. \text { such that } \\
& \left.\zeta_{j}\left(u_{1}, \ldots, u_{j-1}\right) \text { exists and }\left\{\zeta_{j}\left(u_{1}, \ldots, u_{j-1}\right)\right\}=0 \forall 2 \leq j \leq i\right\} .
\end{aligned}
$$

So we have the inclusions

$$
\begin{aligned}
\operatorname{TC}_{\rho} R(\Gamma, G) \subset \cdots & \subset \mathrm{TC}_{\rho}^{i+1} R(\Gamma, G) \\
& \subset \mathrm{TC}_{\rho}^{i} R(\Gamma, G) \subset \cdots \subset \mathrm{QC}_{\rho} R(\Gamma, G) \subset Z^{1}\left(\Gamma, \mathcal{G}_{\rho}\right)
\end{aligned}
$$

and by Artin's theorem, $\operatorname{TC}_{\rho} R(\Gamma, G)=\bigcap_{i \geq 1} \operatorname{TC}_{\rho}^{i} R(\Gamma, G)$.

Definition 2.6 For a given representation $\rho \in R(\Gamma, G)$, we say that $\rho$ is a strongly nonquadratic singularity of $R(\Gamma, G)$ if there exists a cocycle $u_{1} \in \mathrm{QC}_{\rho} R(\Gamma, G)$ in the quadratic cone for which the third obstruction does not vanish. In other words, $\mathrm{TC}_{\rho}^{3} R(\Gamma, G) \varsubsetneqq \mathrm{QC}_{\rho} R(\Gamma, G)$. 
Notation If $G=\mathrm{SL}$ we will adopt the notation $\mathrm{QC}_{\rho}:=\mathrm{QC}_{\rho} R(\Gamma, \mathrm{SL}), \mathrm{TC}_{\rho}^{i}:=$ $\mathrm{TC}_{\rho}^{i} R(\Gamma, \mathrm{SL})$ for $i \geq 3$ and $\mathrm{TC}_{\rho}:=\mathrm{TC}_{\rho} R(\Gamma, \mathrm{SL})$.

\section{Abelian representations of knot groups and the quadratic cone}

From now and on we suppose that $G=\mathrm{SL}$ and $\Gamma$ is a knot group.

In this section we study mainly the Zariski tangent space and the quadratic cone at an abelian representation which corresponds to a root of the Alexander polynomial. In particular, we recall a result of [4] which allows us to calculate explicitly the quadratic cone.

\subsection{Alexander invariants}

Recall that if $\Gamma$ is a knot group then we have a canonical surjection $\Phi: \Gamma \rightarrow T$ where $T:=\Gamma / \Gamma^{\prime}$ is an infinite cyclic group. We denote by $\widetilde{M}$ the infinite cyclic covering determined by the epimorphism $\Phi: \Gamma \rightarrow T \cong \mathbb{Z}$. Let $t$ be a generator of $T$ and let $\Gamma=\left\langle S_{1}, \ldots, S_{n} \mid R_{1}, \ldots, R_{n-1}\right\rangle$ be a presentation of $\Gamma$ such that $\Phi\left(S_{i}\right)=t$. We choose then $\mu:=S_{1}$ as a meridian of $K$.

The vector space $H_{1}(\widetilde{M}, \mathbb{Q})$ is a torsion $\mathbb{Q} T$-module and the group algebra $\Lambda_{0}=\mathbb{Q} T$ can be then identified with the ring of Laurent polynomials $\mathbb{Q}\left[t, t^{-1}\right]$. The module $H_{1}(\widetilde{M}, \mathbb{Q})$ decomposes as a sum of cyclic $\Lambda_{0}$-modules

$$
H_{1}(\widetilde{M}, \mathbb{Q})=\bigoplus_{i=1}^{k} \frac{\Lambda_{0}}{\left(\Pi_{i}\right)} \quad \text { where } \Pi_{i} \in \Lambda_{0} .
$$

The ideals $\left(\Pi_{i}\right)$ satisfy $\left(\Pi_{i}\right) \subset\left(\Pi_{i+1}\right)$, ie $\Pi_{k}\left|\Pi_{k-1} \cdots\right| \Pi_{1}$. The $i$-th Alexander polynomial $\Delta_{i}$ is by definition $\Delta_{i}:=\Pi_{k} \cdots \Pi_{i}$, so $\Pi_{i}=\Delta_{i} / \Delta_{i+1}$.

The first Alexander polynomial $\Delta_{1}$ is the generator of the order ideal of the Alexander module $H_{1}(\widetilde{M}, \mathbb{Q})$. By the Blanchfield duality theorem [5], the Alexander polynomials are symmetric and unique up to multiplication with elements of the form $\left\{\alpha t^{n} \mid \alpha \in\right.$ $\mathbb{Q} \backslash\{0\}, n \in \mathbb{Z}\}$. Note that $\Delta_{i}(1) \neq 0$.

\subsection{Abelian representations}

For a given $\lambda \in \mathbb{C}^{*}$, we denote by $\rho_{\lambda}$ the abelian representation which is given by $\mu \mapsto \operatorname{diag}\left(\lambda, \lambda^{-1}\right) \in \mathrm{SL}$. The Lie algebra $\mathfrak{s l}:=\mathfrak{s l}_{2}(\mathbb{C})$ splits as a $\Gamma$-module

$$
\mathfrak{s l}_{\rho_{\lambda}}=\mathcal{H} \oplus \mathcal{G}_{+} \oplus \mathcal{G}_{-},
$$


where $\mathcal{H}$ is generated by

$$
g_{0}=\left(\begin{array}{cc}
1 & 0 \\
0 & -1
\end{array}\right)
$$

$\mathcal{G}_{+}$is generated by

$$
g_{+}=\left(\begin{array}{ll}
0 & 1 \\
0 & 0
\end{array}\right)
$$

and $\mathcal{G}_{-}$is generated by

$$
g_{-}=\left(\begin{array}{ll}
0 & 0 \\
1 & 0
\end{array}\right) \text {. }
$$

Here $\Gamma$ acts on $\mathcal{G}_{ \pm}$via $\gamma \cdot z_{ \pm}=\Phi(\gamma)\left(\lambda^{ \pm 2}\right) z_{ \pm}$and $\mathcal{H}$ is the trivial $\Gamma$-module. If $f: \Gamma \rightarrow \mathfrak{s l}$ is a cochain, we denote $f^{0}, f^{+}, f^{-}$its coordinates in $g_{0}, g_{+}$and $g_{-}$.

By [18], $H^{1}\left(\Gamma, \mathcal{G}_{ \pm}\right)=0$ if and only if $\Delta_{1}\left(\alpha^{ \pm 1}\right) \neq 0$, where $\alpha:=\lambda^{2}$. It turns out that in this case $\operatorname{dim}_{\mathbb{C}} Z^{1}\left(\Gamma, \mathfrak{s l}_{\rho_{\lambda}}\right)=3, \rho_{\lambda}$ is a smooth point of the representation variety $R(\Gamma)$ and $S(\Gamma)$ is the unique component through $\rho_{\lambda}$ (see Klassen [18]).

Remark The representation $\rho_{\lambda}$ is a singular point of the representation variety $R(\Gamma)$ if $\Delta_{1}(\alpha)=0$. Moreover, it was observed in [3, Corollary 4.4] that the representation $\rho_{\lambda}$ is scheme reduced, so $T_{\rho_{\lambda}}^{\mathrm{Zar}} R(\Gamma)=Z^{1}\left(\Gamma, \mathfrak{s l}_{\rho_{\lambda}}\right)$.

Lemma 3.1 If $u_{1}=W+u_{+}+u_{-} \in Z^{1}(\Gamma, \mathcal{H}) \oplus Z^{1}\left(\Gamma, \mathcal{G}_{+}\right) \oplus Z^{1}\left(\Gamma, \mathcal{G}_{-}\right)$is formally integrable, then so are the cocycles $c u_{1}, c \in \mathbb{C}$ and $\tilde{u}_{1}=W+\beta u_{+}+\beta^{-1} u_{-}, \beta \in \mathbb{C}^{*}$. Moreover, if $u_{1}=u_{+}+u_{-}$is formally integrable then $\hat{u}_{1}=\beta_{+} u_{+}+\beta_{-} u_{-}$is also formally integrable for all $\beta_{ \pm} \in \mathbb{C}$.

Proof Let $\rho_{t}=\exp \left(\sum_{i \geq 1} t^{i} u_{i}\right) \rho_{\lambda}$ be a formal deformation of $\rho_{\lambda}$.

It is clear that $\rho_{t}=\exp \left(\sum_{i \geq 1} c^{i} t^{i} u_{i}\right) \rho_{\lambda}$ is also a formal deformation of $\rho_{\lambda}$ for any $c \in \mathbb{C}$.

On the other hand, the stabilizer of $\rho_{\lambda}$ under conjugation of SL are the diagonal matrices. We have for all $\beta \in \mathbb{C}^{*}$,

$$
\left(\begin{array}{cc}
\beta^{1 / 2} & 0 \\
0 & \beta^{-1 / 2}
\end{array}\right)\left(\begin{array}{cc}
x & y \\
z & -x
\end{array}\right)\left(\begin{array}{cc}
\beta^{-1 / 2} & 0 \\
0 & \beta^{1 / 2}
\end{array}\right)=\left(\begin{array}{cc}
x & \beta y \\
\beta^{-1} z & -x
\end{array}\right),
$$

so the lemma follows for $\tilde{u}_{1}$.

If $\beta_{+} \beta_{-}=0$, then as $\exp \left(t u_{ \pm}\right) \rho_{\lambda}$ is a formal deformation of $\rho_{\lambda}, \widehat{u}_{1}$ is formally integrable. 
If $\beta_{+} \beta_{-} \neq 0$, then the properties above and the equality

$$
\left(\beta_{+}, \beta_{-}\right)=\left(\beta_{+} \beta_{-}\right)^{1 / 2}\left(\beta_{+}^{1 / 2} \beta_{-}^{-1 / 2}, \beta_{+}^{-1 / 2} \beta_{-}^{1 / 2}\right)
$$

show that $\hat{u}_{1}$ is formally integrable.

For $\alpha \in \mathbb{C}$ and a polynomial $\Pi \in \Lambda_{0}$, we denote by $v_{\alpha}(\Pi) \in \mathbb{Z}$ the multiplicity of $\Pi$ at $\alpha$. So $\alpha$ is a root of $\Pi$ if and only if $v_{\alpha}(\Pi)>0$.

Lemma 3.2 Let $\alpha \in \mathbb{C}^{*}$ be a root of $\Delta_{1}$ and set $l=\max \left\{i \mid \Pi_{i}(\alpha)=0\right\}$ where $\Pi_{i}=\Delta_{i} / \Delta_{i+1}$.

Then $\operatorname{dim}_{\mathbb{C}} H^{1}\left(\Gamma, \mathcal{G}_{ \pm}\right)=l$ and $v_{\alpha}\left(\Delta_{1}\right)=\sum_{i=1}^{l} v_{\alpha}\left(\Pi_{i}\right)$.

Proof This is a result of Burde [9] and de Rham [21] and is a consequence of the fact that

$$
H^{1}\left(\Gamma, \mathcal{G}_{ \pm}\right) \cong \operatorname{Hom}\left(H_{1}(\widetilde{M}, \mathbb{C}), \frac{\mathbb{C}\left[t, t^{-1}\right]}{\left(t-\alpha^{ \pm 1}\right)}\right) \text { and } H_{1}(\widetilde{M}, \mathbb{C}) \cong \bigoplus_{i=1}^{k} \frac{\Lambda_{0}}{\left(\Pi_{i}\right)} \otimes \mathbb{C}
$$

\subsection{The quadratic cone}

Let $u_{1}=W+u_{+}+u_{-} \in Z^{1}\left(\Gamma, \mathfrak{s l}_{\rho_{\lambda}}\right)$ be a cocycle where $W \in Z^{1}(\Gamma, \mathcal{H})$ and $u_{ \pm} \in Z^{1}\left(\Gamma, \mathcal{G}_{ \pm}\right)$. The distributive property for the cup product, together with the fact that $[\mathcal{H}, \mathcal{H}]=0=\left[\mathcal{G}_{+}, \mathcal{G}_{+}\right]=\left[\mathcal{G}_{-}, \mathcal{G}_{-}\right]$, give

$u_{1} \smile u_{1}=\left(u_{+} \smile u_{-}+u_{-} \smile u_{+}\right)+\left(W \smile u_{+}+u_{+} \smile W\right)+\left(W \smile u_{-}+u_{-} \smile W\right)$.

Since $H^{2}(\Gamma, \mathcal{H})=0,\left\{u_{1} \smile u_{1}\right\}=\left\{\left(W \smile u_{+}+u_{+} \smile W\right)+\left(W \smile u_{-}+u_{-} \smile W\right)\right\}=$ $2\left\{W \smile u_{+}\right\}+2\left\{W \smile u_{-}\right\}$.

Notice that the cup product is commutative in cohomology because the Lie bracket is anticommutative. It follows that the quadratic cone $\mathrm{QC}_{\rho_{\lambda}}$ contains the space $D^{+}:=Z^{1}(\Gamma, \mathcal{H}) \oplus B^{1}\left(\Gamma, \mathcal{G}_{+}\right) \oplus B^{1}\left(\Gamma, \mathcal{G}_{-}\right)$of cocycles which are cohomologous to diagonal cocycles, and contains the space of antidiagonal cocycles $D^{-}:=Z^{1}\left(\Gamma, \mathcal{G}_{+}\right) \oplus$ $Z^{1}\left(\Gamma, \mathcal{G}_{-}\right)$. It may be worth to notice that $D^{+}$is tangent to the abelian deformations obtained by perturbing $\lambda$.

In fact, the following result of [4, Theorem 3.2] allows us to determine the quadratic cone. 
Proposition 3.3 Let $W \in Z^{1}(\Gamma, \mathcal{H})$ be a nonzero cocycle. The homomorphism

$$
W \smile \cdot: H^{1}\left(\Gamma, \mathcal{G}_{ \pm}\right) \rightarrow H^{2}\left(\Gamma, \mathcal{G}_{ \pm}\right)
$$

is an isomorphism if and only if the $\left(t-\alpha^{ \pm 1}\right)$-torsion of the Alexander module $H_{1}(\widetilde{M}, \mathbb{C})$ is semisimple, that is of the form

$$
\frac{\mathbb{C}\left[t, t^{-1}\right]}{\left(t-\alpha^{ \pm 1}\right)} \oplus \cdots \oplus \frac{\mathbb{C}\left[t, t^{-1}\right]}{\left(t-\alpha^{ \pm 1}\right)} .
$$

Note that the latter is equivalent to the equality $\operatorname{dim}_{\mathbb{C}} H^{1}\left(\Gamma, \mathcal{G}_{ \pm}\right)=v_{\alpha^{ \pm 1}}\left(\Delta_{1}\right)$.

Corollary 3.4 Let $k=\max \left\{i \mid \Pi_{i}(\alpha)=0\right\}$.

(1) If $\operatorname{dim}_{\mathbb{C}} H^{1}\left(\Gamma, \mathcal{G}_{ \pm}\right)=v_{\alpha^{ \pm 1}}\left(\Delta_{1}\right)$, then every cocycle $u \in \mathrm{QC}_{\rho_{\lambda}}$ is an antidiagonal cocycle or cohomologous to a diagonal cocycle, ie $\mathrm{QC}_{\rho_{\lambda}}=D^{+} \cup D^{-}$.

(2) If $v_{\alpha^{ \pm}}\left(\Pi_{i}\right)>1$ for all $i=1, \ldots, k$, then the quadratic cone and the Zariski tangent space coincide, ie $Z^{1}\left(\Gamma, \mathfrak{s l}_{\rho_{\lambda}}\right)=\mathrm{QC}_{\rho_{\lambda}}$.

Proof Let $u_{1}=W+u_{+}+u_{-} \in \mathrm{QC}_{\rho_{\lambda}}$ be a cocycle where $W \in Z^{1}(\Gamma, \mathcal{H}), u_{+} \in$ $Z^{1}\left(\Gamma, \mathcal{G}_{+}\right)$and $u_{-} \in Z^{1}\left(\Gamma, \mathcal{G}_{-}\right)$. We have $u_{1} \smile u_{1}$ is homologous to zero if and only if $W \smile u_{+}$and $W \smile u_{-}$are homologous to zero.

If $\operatorname{dim}_{\mathbb{C}} H^{1}\left(\Gamma, \mathcal{G}_{ \pm}\right)=v_{\alpha^{ \pm 1}}\left(\Delta_{1}\right)$ then, by Proposition 3.3, we obtain $W=0$ or $u_{ \pm} \in$ $B^{1}\left(\Gamma, \mathcal{G}_{ \pm}\right)$.

If $v_{\alpha^{ \pm}}\left(\Pi_{i}\right)>1$ for all $i=1, \ldots, k$, then $W \smile u_{+}$and $W \smile u_{-}$are homologous to zero (see [4, proof of Theorem 3.2]) and hence $Z^{1}\left(\Gamma, \mathfrak{s l}_{\rho_{\lambda}}\right)=\mathrm{QC}_{\rho_{\lambda}}$.

Now, Theorem 5.2 of [3] can be rephrased as follows:

Corollary 3.5 Let $K$ be a knot in $S^{3}$ with group $\Gamma$. Let $\lambda \in \mathbb{C}^{*}$ such that $\lambda^{2}$ is a simple root of the Alexander polynomial and $\rho_{\lambda}$ the abelian representation which is given by $\mu \mapsto \operatorname{diag}\left(\lambda, \lambda^{-1}\right) \in \mathrm{SL}$. Then the representation variety $R(\Gamma)$ has a quadratic singularity at $\rho_{\lambda}$, ie

$$
\mathrm{TC}_{\rho_{\lambda}}=\mathrm{QC}_{\rho_{\lambda}} \varsubsetneqq Z^{1}\left(\Gamma, \mathfrak{s l}_{\rho_{\lambda}}\right) .
$$

The following result asserts the existence of knots with nonquadratic singularities.

Theorem 3.6 Let $K \subset S^{3}$ be a knot and let $\lambda \in \mathbb{C}^{*}$ such that the $(t-\alpha)$-torsion of the Alexander module of $K$ with complex coefficients is cyclic of the form

$$
\frac{\mathbb{C}\left[t, t^{-1}\right]}{\left(t-\lambda^{2}\right)^{r}} \oplus \frac{\mathbb{C}\left[t, t^{-1}\right]}{\left(t-\lambda^{-2}\right)^{r}}, \quad r \geq 2 .
$$

Then the representation variety $R(\Gamma)$ has a nonquadratic singularity at $\rho_{\lambda}$, where $\rho_{\lambda}$ is the abelian representation which is given by $\mu \mapsto \operatorname{diag}\left(\lambda, \lambda^{-1}\right) \in \mathrm{SL}$. 
Proof The tangent space $Z^{1}\left(\Gamma, \mathfrak{s l}_{\rho_{\lambda}}\right)$ and the quadratic cone $\mathrm{QC}_{\rho_{\lambda}}$ coincide by Corollary 3.4. Since the $(t-\alpha)$-torsion of the Alexander module of $K$ is cyclic, we have $\operatorname{dim}_{\mathbb{C}} H^{1}\left(\Gamma, \mathcal{G}_{ \pm}\right)=1$ and hence $\operatorname{dim}_{\mathbb{C}} Z^{1}\left(\Gamma, \mathfrak{s l}_{\rho_{\lambda}}\right)=5$.

On the other hand, it is proved in [17, Lemma 6.1] that there are no 5-dimensional components going through $\rho_{\lambda}$. Hence there are cocycles in the quadratic cone which do not appear in the tangent cone.

Remarks (1) Theorem 1.1 allows us to determine a family of knots such that the corresponding representation variety $R(\Gamma)$ admits a strongly nonquadratic singularity. Namely, every knot which has Alexander module of the form $\Lambda /(t-\alpha)^{2} \oplus$ $\Lambda /\left(t-\alpha^{-1}\right)^{2}$ admits a strongly nonquadratic singularity.

(2) One way of constructing a family of knots of $S^{3}$ admitting strongly nonquadratic singularities (and thus nonquadratic singularities) was communicated to me by M Heusener. It corresponds to the product knots $K \sharp K$ where $K$ is a knot in $S^{3}$ such that $\alpha$ is a simple root of the Alexander polynomial $\Delta_{1}$ of $K$.

(3) It is to note that knots having Alexander module of the form $\Lambda /(t-\alpha)^{r} \oplus$ $\Lambda /\left(t-\alpha^{-1}\right)^{r}, r \geq 3$, admit nonquadratic singularities but no strongly nonquadratic singularities.

\section{The third obstruction}

This section is devoted to the proof of the Theorem 1.1 and is divided into four subsections. In the first one we recall the construction of the rationalized group and the involution on the knot group. In the second one we construct an appropriate basis of the first and second homological groups corresponding to the abelian representation $\rho_{\lambda}$. At the end of this subsection, we calculate the space of cocycles corresponding to a reducible nonabelian representation with the same character as $\rho_{\lambda}$. The third and fourth subsections contain the proof of Theorem 1.1.

In this section we will assume that $\alpha:=\lambda^{2}$ is a root of the Alexander polynomial and that the $(t-\alpha)$-torsion of the Alexander module is cyclic of the form $\Lambda /(t-\alpha)^{r} \oplus$ $\Lambda /\left(t-\alpha^{-1}\right)^{r}$ where $\Lambda:=\mathbb{C}\left[t, t^{-1}\right]$ and $r$ is a positive integer.

By Corollary 3.5, if $r=1$ then $\mathrm{TC}_{\rho_{\lambda}}=\mathrm{QC}_{\rho_{\lambda}} \varsubsetneqq Z^{1}\left(\Gamma, \mathfrak{s l}_{\rho_{\lambda}}\right)$. In particular, $\mathrm{TC}_{\rho_{\lambda}}=$ $\mathrm{TC}_{\rho_{\lambda}}^{3}=\mathrm{QC}_{\rho_{\lambda}}$.

Our aim is to determine the cubic tangent cone at $\rho_{\lambda}$ to $R(\Gamma)$ as an approach to the tangent cone $\mathrm{TC}_{\rho_{\lambda}}$.

As a consequence of Theorem 1.1, we observe that the third obstruction vanishes if $r \geq 3$. So our studies suggest the following conjecture: 
Conjecture If $\alpha$ is a root of the Alexander polynomial such that the $(t-\alpha)$-torsion of the Alexander module is of the form $\Lambda /(t-\alpha)^{r} \oplus \Lambda /\left(t-\alpha^{-1}\right)^{r}, r \geq 1$

then

$$
\mathrm{TC}_{\rho_{\lambda}}=\mathrm{TC}_{\rho_{\lambda}}^{r+1} \varsubsetneqq \mathrm{TC}_{\rho_{\lambda}}^{r}=\cdots=Z^{1}\left(\Gamma, \mathfrak{s l}_{\rho_{\lambda}}\right) .
$$

This conjecture is verified for $r=1$ and $r=2$. For $r \geq 3$, we just proved that $\mathrm{TC}_{\rho_{\lambda}}^{3}=\mathrm{QC}_{\rho_{\lambda}}=Z^{1}\left(\Gamma, \mathfrak{s l}_{\rho_{\lambda}}\right)$. Moreover, by Proposition 4.1, $W+V_{+} \in \mathrm{TC}_{\rho_{\lambda}}^{r}$. So, we project to prove that $W+V_{+} \notin \mathrm{TC}_{\rho_{\lambda}}^{r+1}$ as a first step in proving the conjecture.

The proof of Theorem 1.1 will be divided into two parts. The first step consists in the choice of an antidiagonal, nontrivial cocycle $u_{1} \in Z^{1}\left(\Gamma, \mathcal{G}_{-} \oplus \mathcal{G}_{+}\right)$. Using the techniques of deformation of [4], we will construct in the case where $r=2$ and $u_{1}$ is a cocycle which verifies the third obstruction, a formal deformation tangent to $u_{1}$. In the case where the third obstruction does not vanish for $u_{1}$ and $r \geq 2$ we will use a result of [16] to conclude.

\subsection{The rationalized group and involution}

Our basic reference in this paragraph is [4], all proofs and details can be found there. However, for the sake of completeness, we recall the following constructions in order to solve the obstruction equations.

Let $K$ be a knot in $S^{3}$. Since the Alexander polynomials are reciprocal, the $\Lambda_{0}-$ module $H_{1}(\widetilde{M}, \mathbb{Q})$ decomposes as a direct sum of modules of the form $\Lambda_{0} / p^{m}(t)$ and $\Lambda_{0} / q^{n}(t) \oplus \Lambda_{0} / q^{n}\left(t^{-1}\right)$, where $p(t)$ is an irreducible reciprocal polynomial, $q(t)$ is an irreducible nonreciprocal polynomial and $m, n$ are nonnegative integers.

Construct an anti-involution $j$ on $H_{1}(\widetilde{M}, \mathbb{Q})$ by setting

$$
\left\{\begin{array}{clrl}
j([R(t)]) & =R\left(\left[t^{-1}\right]\right) & & \text { on } \frac{\Lambda_{0}}{p^{m}(t)} \\
j\left(\left[S^{1}(t)\right],\left[S^{2}(t)\right]\right) & =\left(\left[S^{2}\left(t^{-1}\right)\right],\left[S^{1}\left(t^{-1}\right)\right]\right) & & \text { on } \frac{\Lambda_{0}}{q^{n}(t)} \oplus \frac{\Lambda_{0}}{q^{n}\left(t^{-1}\right)} .
\end{array}\right.
$$

This anti-involution extends to $H_{1}(\widetilde{M}, \mathbb{C})$ to an anti-involution still denoted $j$. In particular, if we suppose that the $(t-\alpha)$-torsion of the $\Lambda$-module $H_{1}(\widetilde{M}, \mathbb{C})$ is isomorphic to $\Lambda /(t-\alpha)^{r} \oplus \Lambda /\left(t-\alpha^{-1}\right)^{r}$ then $j\left(\left[Y_{1}(t)\right],\left[Y_{2}(t)\right]\right)=\left(\left[Y_{2}\left(t^{-1}\right)\right],\left[Y_{1}\left(t^{-1}\right)\right]\right)$.

Let $N=N_{1}=\Gamma^{\prime}$ be the commutator subgroup of $\Gamma=\pi_{1}(M), N_{i+1}=\left[N, N_{i}\right]$ and let $\widehat{\Gamma}=\widehat{N / N}_{i} \rtimes T$ be the rationalized associated group as constructed in [4]. Then the anti-involution $j$ extends to an involution on $\widehat{\Gamma}$ and induce involutions in cochains and in cohomology which we simply denote $j^{*}$. 
By restricting ourselves to a subcomplex of the standard cochain complex $C^{*}\left(\widehat{\Gamma}, \mathfrak{s l}_{\rho_{\lambda}}\right)$, we obtain the cochain complex $\overline{C^{*}}\left(\widehat{\Gamma}, \mathfrak{s l}_{\rho_{\lambda}}\right)$ which is isomorphic to the direct limit $\left.\lim _{\longrightarrow} C^{*}\left(\widehat{\left(N / N_{i}\right.}\right) \rtimes T, \mathfrak{s l}_{\rho_{\lambda}}\right)$ and whose cohomology groups are the same as those of $\Gamma$

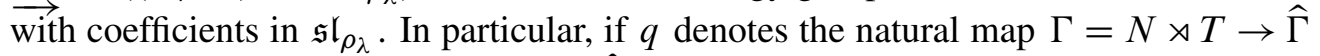
then $q$ induces isomorphisms $q^{*}: \overline{H^{*}}\left(\hat{\Gamma}, \mathcal{G}_{ \pm}\right) \rightarrow H^{*}\left(\Gamma, \mathcal{G}_{ \pm}\right)$for $i=1,2$, (for details see [4, Section 3, proof of Proposition 3.1]).

Remark It is to notice that $\partial \circ j^{*}=j^{*} \circ \partial$ and that, for all $f, g \in \overline{Z^{1}}\left(\widehat{\Gamma}, \mathfrak{s l}_{\rho_{\lambda}}\right)$, $j^{*}(f \smile g)=-j^{*} f \smile j^{*} g[2$, Chapter III].

\subsection{Construction of an appropriate basis of $H^{1}\left(\Gamma, \mathcal{G}_{+} \oplus \mathcal{G}_{-}\right)$and $H^{2}\left(\Gamma, \mathcal{G}_{+} \oplus \mathcal{G}_{-}\right)$}

As $\alpha$ is a root of the Alexander polynomial, we have an isomorphism [4]

$\bar{\psi}: \operatorname{Hom}_{\Lambda}\left(H_{1}(\widetilde{M}, \mathbb{C}), \mathcal{G}_{+} \oplus \mathcal{G}_{-}\right) \rightarrow \overline{H^{1}}\left(\widehat{\Gamma}, \mathcal{G}_{+} \oplus \mathcal{G}_{-}\right) \cong H^{1}\left(\Gamma, \mathcal{G}_{+} \oplus \mathcal{G}_{-}\right)$.

Let $y_{1}$ be a $\Lambda$-basis of the $(t-\alpha)$-torsion of $H_{1}(\widetilde{M}, \mathbb{C}), \Lambda /(t-\alpha)^{r}$, and set $y_{2}=$ $j\left(y_{1}\right)$. Let $\left(V_{+}, V_{-}\right)$be cocycles in $\overline{Z^{1}}\left(\widehat{\Gamma}, \mathcal{G}_{+} \oplus \mathcal{G}_{-}\right)$such that

$$
\left\{\begin{array}{l}
\bar{\psi}^{-1}\left(\left\{V_{+}\right\}\right)\left(y_{1}\right)=g_{+} \\
\bar{\psi}^{-1}\left(\left\{V_{-}\right\}\right)\left(y_{2}\right)=g_{-} .
\end{array}\right.
$$

Then $\left\{V_{+}\right\}$(resp. $\left.\left\{V_{-}\right\}\right)$is a basis of $\overline{H^{1}}\left(\widehat{\Gamma}, \mathcal{G}_{+}\right)\left(\right.$resp. $\left.\overline{H^{1}}\left(\widehat{\Gamma}, \mathcal{G}_{-}\right)\right)$such that $j^{*}\left(V_{+}\right)=$ $V_{-}$.

We will use later the following result whose proof will be postponed to Section 6 . One can show that this result, which provides us a basis of $H^{2}\left(\Gamma, \mathcal{G}_{+}\right)$, may be generalized to the case where the torsion of $H_{1}(\widetilde{M}, \mathbb{C})$ is arbitrary, but we will restrict ourselves to the special case where the torsion is cyclic.

Proposition 4.1 If $H_{1}(\widetilde{M}, \mathbb{C})$ is a cyclic module with $(t-\alpha)$-torsion $\Lambda /(t-\alpha)^{r}$, $r \geq 2$, and $W \in Z^{1}(\Gamma, \mathcal{H})$ a nonzero cocycle, then there exist cochains $h_{i}^{+} \in C^{1}\left(\Gamma, \mathcal{G}_{+}\right)$, $2 \leq i \leq r$ such that $\left\{\zeta_{r+1}\left(W+V_{+}, h_{2}^{+}, \ldots, h_{r}^{+}\right)\right\}$is a basis of $H^{2}\left(\Gamma, \mathcal{G}_{+}\right)$.

The proof shows that the cochains $h_{i}^{+}$factor through $N_{2}$ and define cochains from $\widehat{\Gamma}$ to $\mathcal{G}_{+}$still denoted by $h_{i}^{+}$.

Corollary 4.2 Let the cochains $h_{i}^{-}:=j^{*} h_{i}^{+} \in \overline{C^{1}}\left(\widehat{\Gamma}, \mathcal{G}_{-}\right), 2 \leq i \leq r$. Then $\left\{\zeta_{r+1}\left(W+V_{-}, h_{2}^{-}, \ldots, h_{r}^{-}\right)\right\}$is a basis of $\overline{H^{2}}\left(\widehat{\Gamma}, \mathcal{G}_{-}\right)$.

In fact the cochains $h_{i}^{-}$are defined on $\Gamma$. 
The cocycle $U_{1}=V_{+}+V_{-}$satisfies $j^{*} U_{1}=U_{1}$.

Consider the second obstruction equation

$$
-\partial U_{2}=\zeta\left(U_{1}\right)=\frac{1}{2} U_{1} \smile U_{1} .
$$

Then there exists a cochain $U_{2} \in \overline{C^{1}}(\widehat{\Gamma}, \mathcal{H})$ which solves $\left(E_{2}\right)$ and verifies $U_{2}(\mu)=0$. Applying the involution $j^{*}$ to the obstruction equation $\left(E_{2}\right)$ [3, Section 4.1], one obtains

$$
-\partial\left(j^{*} U_{2}\right)=-\frac{1}{2} U_{1} \smile U_{1}=\partial U_{2} .
$$

So $j^{*} U_{2}=-U_{2}+Z$ where $Z \in \overline{Z^{1}}(\hat{\Gamma}, \mathcal{H})$. As $\left(j^{*} U_{2}\right)(\mu)=U_{2}\left(\mu^{-1}\right)=0=U_{2}(\mu)$, $Z(\mu)=0$ and $Z=0$. Thus $j^{*} U_{2}=-U_{2}$.

Consider now the $2-$ cocycle $\zeta_{3}\left(U_{1}, U_{2}\right) \in \overline{Z^{2}}\left(\widehat{\Gamma}, \mathcal{G}_{+} \oplus \mathcal{G}_{-}\right)$; then $\left\{\zeta_{3}\left(U_{1}, U_{2}+Z\right)\right\}=$ $\left\{\zeta_{3}\left(U_{1}, U_{2}\right)\right\}+\{W \cup Z\}$ for all $Z \in \overline{Z^{1}}\left(\widehat{\Gamma}, \mathfrak{s l}_{\rho_{\lambda}}\right)$.

By Corollary 3.4 (2), as $r \geq 2$,

$\left\{\zeta_{3}\left(U_{1}, U_{2}\right)\right\}=0$ in $\overline{H^{2}}\left(\widehat{\Gamma}, \mathfrak{s l}_{\rho_{\lambda}}\right)$ if and only if

$$
\left\{\zeta_{3}\left(U_{1}, U_{2}+Z\right)\right\}=0 \text { in } \overline{H^{2}}\left(\widehat{\Gamma}, \mathfrak{s l}_{\rho_{\lambda}}\right) \text { for all } Z \in \overline{Z^{1}}\left(\widehat{\Gamma}, \mathfrak{s l}_{\rho_{\lambda}}\right) .
$$

Notation In the sequel we let $\phi_{\lambda}: \Gamma \rightarrow$ SL be the reducible nonabelian representation given by $\phi_{\lambda}=\exp \left(V_{+}\right) \rho_{\lambda}$.

Lemma 4.3 Suppose that $r=2$. Then

$$
\operatorname{dim}_{\mathbb{C}} Z^{1}\left(\Gamma, \mathfrak{s l}_{\phi_{\lambda}}\right)= \begin{cases}4 & \text { if }\left\{\zeta_{3}\left(U_{1}, U_{2}\right)\right\} \neq 0 \text { in } H^{2}\left(\Gamma, \mathfrak{s l}_{\rho_{\lambda}}\right) \\ 5 & \text { if }\left\{\zeta_{3}\left(U_{1}, U_{2}\right)\right\}=0 \text { in } H^{2}\left(\Gamma, \mathfrak{s l}_{\rho_{\lambda}}\right)\end{cases}
$$

More precisely, let $W \in Z^{1}(\Gamma, \mathbb{C})$ be a cocycle such that

$$
W(\mu)=\left(\begin{array}{cc}
1 & 0 \\
0 & -1
\end{array}\right) \text {. }
$$

Then, with the same notation as above we obtain:

(1) if $\left\{\zeta_{3}\left(U_{1}, U_{2}\right)\right\} \neq 0$ in $H^{2}\left(\Gamma, \mathfrak{s l}_{\rho_{\lambda}}\right)$ then

$$
Z^{1}\left(\Gamma, \mathfrak{s l}_{\phi_{\lambda}}\right)=B^{1}\left(\Gamma, \mathfrak{s l}_{\phi_{\lambda}}\right) \oplus\left\langle\left(W+h_{2}^{+}-\frac{1}{2}\left[W, V_{+}\right]\right)\right\rangle .
$$


(2) if $\left\{\zeta_{3}\left(U_{1}, U_{2}\right)\right\}=0$ in $H^{2}\left(\Gamma, \mathfrak{s l}_{\rho_{\lambda}}\right)$ and $U_{3} \in C^{1}\left(\Gamma, \mathcal{G}_{+} \oplus \mathcal{G}_{-}\right)$is a cochain such that $-\partial U_{3}=\zeta_{3}\left(U_{1}, U_{2}\right)$ then

$$
\begin{aligned}
Z^{1}\left(\Gamma, \mathfrak{s l}_{\phi_{\lambda}}\right)= & B^{1}\left(\Gamma, \mathfrak{s l}_{\phi_{\lambda}}\right) \oplus\left\langle\left(W+h_{2}^{+}-\frac{1}{2}\left[W, V_{+}\right]\right)\right\rangle \\
& \oplus\left\langle\left(V_{-}+U_{2}+\frac{1}{2}\left[V_{+}, V_{-}\right]+U_{3}^{+}+\frac{1}{2}\left[V_{+}, U_{2}\right]-\frac{1}{6}\left[V_{+},\left[V_{+}, V_{-}\right]\right]\right)\right\rangle .
\end{aligned}
$$

Proof Let $\mathcal{U} \in Z^{1}\left(\Gamma, \mathfrak{s l}_{\phi_{\lambda}}\right)$ be a cocycle; then for all $\gamma_{1}, \gamma_{2} \in \Gamma$

$$
\mathcal{U}\left(\gamma_{1} \gamma_{2}\right)=\mathcal{U}\left(\gamma_{1}\right)+\phi_{\lambda}\left(\gamma_{1}\right) \mathcal{U}\left(\gamma_{2}\right) \phi_{\lambda}^{-1}\left(\gamma_{1}\right)
$$

Now, using the decomposition $\mathcal{U}=\mathcal{U}^{0}+\mathcal{U}^{+}+\mathcal{U}^{-}$, where $\mathcal{U}^{ \pm}$takes values in $\mathcal{G}^{ \pm}$ and $\mathcal{U}^{0}$ in $\mathcal{H}$, one obtains that, for all $\gamma_{1}, \gamma_{2} \in \Gamma,(*)$ is equivalent to the system

$$
\left\{\begin{aligned}
\mathcal{U}^{-}\left(\gamma_{1} \gamma_{2}\right) & =\mathcal{U}^{-}\left(\gamma_{1}\right)+\gamma_{1} \cdot \mathcal{U}^{-}\left(\gamma_{2}\right) \\
\mathcal{U}^{0}\left(\gamma_{1} \gamma_{2}\right) & =\mathcal{U}^{0}\left(\gamma_{1}\right)+\mathcal{U}^{0}\left(\gamma_{2}\right)+\left[V_{+}\left(\gamma_{1}\right), \gamma_{1} \cdot \mathcal{U}^{-}\left(\gamma_{2}\right)\right] \\
\mathcal{U}^{+}\left(\gamma_{1} \gamma_{2}\right) & =\mathcal{U}^{+}\left(\gamma_{1}\right)+\gamma_{1} \cdot \mathcal{U}^{+}\left(\gamma_{2}\right)+\left[V_{+}\left(\gamma_{1}\right), \mathcal{U}^{0}\left(\gamma_{2}\right)\right] \\
& \quad+\frac{1}{2}\left[V_{+}\left(\gamma_{1}\right),\left[V_{+}\left(\gamma_{1}\right), \gamma_{1} \cdot \mathcal{U}^{-}\left(\gamma_{2}\right)\right]\right]
\end{aligned}\right.
$$

which is equivalent to

$$
\left\{\begin{aligned}
\mathcal{U}^{-} & \in Z^{1}\left(\Gamma, \mathcal{G}_{-}\right) \\
-\partial \mathcal{U}^{0} & =V_{+} \smile \mathcal{U}^{-} \\
-\partial \mathcal{U}^{+}\left(\gamma_{1}, \gamma_{2}\right) & =V_{+} \smile \mathcal{U}^{0}\left(\gamma_{1}, \gamma_{2}\right)+\frac{1}{2}\left[V_{+}\left(\gamma_{1}\right), V_{+} \smile \mathcal{U}_{-}\left(\gamma_{1}, \gamma_{2}\right)\right] .
\end{aligned}\right.
$$

Note that if $\mathcal{U}^{-}=0$ then $\mathcal{U}^{0} \in Z^{1}(\Gamma, \mathcal{H})$ and if $\mathcal{U}^{-}=0$ and $\mathcal{U}^{0}=0$ then $\mathcal{U}^{+} \in$ $Z^{1}\left(\Gamma, \mathcal{G}_{+}\right)$. On the other hand, as $\operatorname{dim}_{\mathbb{C}} Z^{1}\left(\Gamma, \mathcal{G}_{ \pm}\right)=2$ and $\operatorname{dim}_{\mathbb{C}} Z^{1}(\Gamma, \mathcal{H})=1$, one obtains $\operatorname{dim}_{\mathbb{C}} Z^{1}\left(\Gamma, \mathfrak{s l}_{\phi_{\lambda}}\right) \leq 5$. Observe further that the space of coboundaries, $B^{1}\left(\Gamma, \mathfrak{s l}_{\phi_{\lambda}}\right)$, is three dimensional and is spanned by the following three elements $v_{i}=\left(\mathcal{U}_{i}^{-}, \mathcal{U}_{i}^{0}, \mathcal{U}_{i}^{+}\right): \Gamma \rightarrow \mathfrak{s l}:$

$$
v_{1}=\left(0,0, \partial B_{+}\right), \quad v_{2}=\left(0,0, V_{+}\right), \quad v_{3}=\left(\partial B_{-}, *, *\right), \quad B_{ \pm} \in \mathcal{G}_{ \pm} .
$$

A simple cohomological calculation enables us to show that, when $U_{3}$ exists, the cochains $v_{4}, v_{5}: \Gamma \rightarrow \mathfrak{s l}$ given, respectively, by

$$
\left\{\begin{array} { l } 
{ \mathcal { U } ^ { - } = 0 } \\
{ \mathcal { U } ^ { 0 } = W } \\
{ \mathcal { U } ^ { + } = h _ { 2 } ^ { + } - \frac { 1 } { 2 } [ W , V _ { + } ] }
\end{array} \quad \left\{\begin{array}{l}
\mathcal{U}^{-}=V_{-} \\
\mathcal{U}^{0}=U_{2}+\frac{1}{2}\left[V_{+}, V_{-}\right] \\
\mathcal{U}^{+}=U_{3}^{+}+\frac{1}{2}\left[V_{+}, U_{2}\right]-\frac{1}{6}\left[V_{+},\left[V_{+}, V_{-}\right]\right]
\end{array}\right.\right.
$$


are linearly independent solutions of the preceding system which are not coboundaries. When $\left\{\zeta_{3}\left(U_{1}, U_{2}\right)\right\} \neq 0$ in $H^{2}\left(\Gamma, \mathfrak{s l}_{\rho_{\lambda}}\right), v_{4}$ is the unique solution of the system which is not a coboundary.

\subsection{The case where $r=2$ and $\left\{\zeta_{3}\left(U_{1}, U_{2}\right)\right\}=0$ in $\left.H^{2}\left(\Gamma, \mathfrak{s l}_{\rho_{\lambda}}\right)\right)$}

In this case we will construct explicitly a formal deformation of the abelian representation $\rho_{\lambda}$ by resolving all the obstruction equations (for more details see Ben Abdelghani and Lines [4]).

As $\left\{\zeta_{3}\left(U_{1}, U_{2}\right)\right\}=0$ in $\overline{H^{2}}\left(\widehat{\Gamma}, \mathfrak{s l}_{\rho_{\lambda}}\right)$, we choose a cochain $U_{3} \in \overline{C^{1}}\left(\widehat{\Gamma}, \mathcal{G}_{+} \oplus \mathcal{G}_{-}\right)$ such that

$$
\left\{\begin{aligned}
-\partial U_{3} & =\zeta_{3}\left(U_{1}, U_{2}\right) \\
j^{*} U_{3} & =U_{3} .
\end{aligned}\right.
$$

This is always possible. Indeed, if $j^{*} U_{3}=U_{3}$ we are done, if not we choose $U_{3}^{0}$ any cochain such that $-\partial U_{3}^{0}=\zeta_{3}\left(U_{1}, U_{2}\right)$ and we set $U_{3}=\frac{1}{2}\left(U_{3}^{0}+j^{*}\left(U_{3}^{0}\right)\right)$.

Now, $\left\{\zeta_{4}\left(U_{1}, U_{2}, U_{3}\right)\right\}=0$ in $\overline{H^{2}}(\widehat{\Gamma}, \mathcal{H})$ and there exists a cochain $U_{4} \in \overline{C^{1}}(\widehat{\Gamma}, \mathcal{H})$ such that

$$
\left\{\begin{array}{l}
-\partial U_{4}=\zeta_{4}\left(U_{1}, U_{2}, U_{3}\right) \\
U_{4}(\mu)=0 .
\end{array}\right.
$$

Using the properties of the involution as previously, it is simple to see that $j^{*} U_{4}=-U_{4}$. The 2-cocycle $\zeta_{5}\left(U_{1}, U_{2}, U_{3}, U_{4}\right)$ may not be cohomologous to zero in $\overline{H^{2}}\left(\widehat{\Gamma}, \mathfrak{s l}_{\rho_{\lambda}}\right)$, so we will perturb the solution $U_{2}$ by a trivial cocycle and study the changes on the related cochains.

Let $\beta \in \mathbb{C}$; then for each cocycle $W \in Z^{1}(\Gamma, \mathcal{H})$ such that

$$
W(\mu)=\left(\begin{array}{cc}
1 & 0 \\
0 & -1
\end{array}\right) \text {, }
$$

the cochain $U_{2}+\beta W$ solves $\left(E_{2}\right)$ and verifies $j^{*}\left(U_{2}+\beta W\right)=-\left(U_{2}+\beta W\right)$. The cochain $U_{3}+\beta\left(h_{2}^{+}+h_{2}^{-}\right)$is then a solution of $\left(E_{3}\right)$ such that $j^{*}\left(U_{3}+\beta\left(h_{2}^{+}+h_{2}^{-}\right)\right)=$ $U_{3}+\beta\left(h_{2}^{+}+h_{2}^{-}\right)$and

$$
\zeta_{4}\left(U_{1}, U_{2}+\beta W, U_{3}+\beta\left(h_{2}^{+}+h_{2}^{-}\right)\right)=\zeta_{4}\left(U_{1}, U_{2}, U_{3}\right)+\beta \eta_{4}
$$

where, for all $\gamma_{1}, \gamma_{2} \in \widehat{\Gamma}$,

$$
\begin{aligned}
\eta_{4}\left(\gamma_{1}, \gamma_{2}\right)= & \frac{1}{2} U_{1} \smile\left(h_{2}^{+}+h_{2}^{-}\right)\left(\gamma_{1}, \gamma_{2}\right)+\frac{1}{2}\left(h_{2}^{+}+h_{2}^{-}\right) \smile U_{1}\left(\gamma_{1}, \gamma_{2}\right) \\
& +\frac{1}{12}\left[\left[U_{1}\left(\gamma_{1}\right), \gamma_{1} \cdot W\left(\gamma_{2}\right)\right]+\left[W\left(\gamma_{1}\right), \gamma_{1} \cdot U_{1}\left(\gamma_{2}\right)\right], \gamma_{1} \cdot U_{1}\left(\gamma_{2}\right)-U_{1}\left(\gamma_{1}\right)\right]
\end{aligned}
$$


As $\eta_{4} \in \overline{Z^{2}}(\widehat{\Gamma}, \mathcal{H})$, there exists a cochain $Z_{4} \in \overline{C^{1}}(\widehat{\Gamma}, \mathcal{H})$ such that

$$
\left\{\begin{array}{l}
-\partial Z_{4}=\eta_{4} \\
Z_{4}(\mu)=0 .
\end{array}\right.
$$

Using again the properties of the involution, one can prove that $j^{*} Z_{4}=-Z_{4}$.

Then a simple calculation gives

$$
\begin{aligned}
\zeta_{5}\left(U_{1}, U_{2}\right. & \left.+\beta W, U_{3}+\beta\left(h_{2}^{+}+h_{2}^{-}\right), U_{4}+\beta Z_{4}\right) \\
& =\zeta_{5}\left(U_{1}, U_{2}, U_{3}, U_{4}\right)+\beta \eta_{5}+\beta^{2}\left(\zeta_{3}\left(W+V_{+}, h_{2}^{+}\right)+\zeta_{3}\left(W+V_{-}, h_{2}^{-}\right)\right),
\end{aligned}
$$

where $\eta_{5} \in \overline{Z^{2}}\left(\widehat{\Gamma}, \mathcal{G}_{+} \oplus \mathcal{G}_{-}\right)$and is such that $j^{*} \eta_{5}=\eta_{5}$.

By Proposition 4.1, there exist two tuples $\left(\delta, \delta^{\prime}\right),\left(\lambda_{5}, \lambda_{5}^{\prime}\right) \in \mathbb{C}^{2}$ such that

$$
\left\{\eta_{5}\right\}=\delta\left\{\zeta_{3}\left(W+V_{1}, h_{2}^{+}\right)\right\}+\delta^{\prime}\left\{\zeta_{3}\left(W+V_{2}, h_{2}^{-}\right)\right\}
$$

and $\quad\left\{\zeta_{5}\left(U_{1}, U_{2}, U_{3}, U_{4}\right)\right\}=\lambda_{5}\left\{\zeta_{3}\left(W+V_{1}, h_{2}^{+}\right)\right\}+\lambda_{5}^{\prime}\left\{\zeta_{3}\left(W+V_{2}, h_{2}^{-}\right)\right\}$.

Using the techniques of [3, Proposition 4.7], one can show $\delta=\delta^{\prime}$ and $\lambda_{5}=\lambda_{5}^{\prime}$. So

$$
\left\{\eta_{5}\right\}=\delta\left\{\zeta_{3}\left(W+V_{1}, h_{2}^{+}\right)+\zeta_{3}\left(W+V_{2}, h_{2}^{-}\right)\right\}
$$

and

$$
\left\{\zeta_{5}\left(U_{1}, U_{2}, U_{3}, U_{4}\right)\right\}=\lambda_{5}\left\{\zeta_{3}\left(W+V_{1}, h_{2}^{+}\right)+\zeta_{3}\left(W+V_{2}, h_{2}^{-}\right)\right\} \text {. }
$$

On the other hand,

$$
\begin{aligned}
& \left\{\zeta_{5}\left(U_{1}, U_{2}+\beta W, U_{3}+\beta\left(h_{2}^{+}+h_{2}^{-}\right), U_{4}+\beta Z_{4}\right)\right\} \\
& =\left\{\zeta_{5}\left(U_{1}, U_{2}, U_{3}, U_{4}\right)\right\}+\left(\beta \delta+\beta^{2}\right)\left\{\zeta_{3}\left(W+V_{+}, h_{2}^{+}\right)+\zeta_{3}\left(W+V_{-}, h_{2}^{-}\right)\right\} \\
& =\left(\beta^{2}+\beta \delta+\lambda_{5}\right)\left\{\zeta_{3}\left(W+V_{+}, h_{2}^{+}\right)+\zeta_{3}\left(W+V_{-}, h_{2}^{-}\right)\right\} .
\end{aligned}
$$

By these equalities and as $\left\{\zeta_{3}\left(W+V_{+}, h_{2}^{ \pm}\right)\right\} \neq 0$ in $\overline{H^{2}}\left(\widehat{\Gamma}, \mathcal{G}_{ \pm}\right)$, we can see that there exist cochains, which we will denote $U_{2}^{0}, U_{3}^{0}, U_{4}^{0}$, such that $\left\{\zeta_{5}\left(U_{1}, U_{2}^{0}, U_{3}^{0}, U_{4}^{0}\right)\right\} \neq 0$ in $\overline{H^{2}}\left(\widehat{\Gamma}, \mathfrak{s l}_{\rho_{\lambda}}\right)$. Then, we can assert that there exists $\lambda_{5} \in \mathbb{C}^{*}$ such that

$$
\left\{\zeta_{5}\left(U_{1}, U_{2}^{0}, U_{3}^{0}, U_{4}^{0}\right)\right\}=\lambda_{5}\left(\left\{\zeta_{3}\left(W+V_{+}, h_{2}^{+}\right)+\zeta_{3}\left(W+V_{-}, h_{2}^{-}\right)\right\}\right) .
$$

Note that, if $\beta$ is a solution of the equation $\beta^{2}+\beta \delta+\lambda_{5}=0$ then $\beta \neq 0$. So, by the equalities above, we can always manage to have two distinct possible choices $\beta_{0} \in \mathbb{C}^{*}$ for which

$$
\left\{\zeta_{5}\left(U_{1}, U_{2}^{0}+\beta_{0} W, U_{3}^{0}+\beta_{0}\left(h_{2}^{+}+h_{2}^{-}\right), U_{4}^{0}+\beta_{0} Z_{4}\right)\right\}=0 \quad \text { in } \overline{H^{2}}\left(\widehat{\Gamma}, \mathfrak{s l}_{\rho_{\lambda}}\right) .
$$


We denote $U_{2}:=U_{2}^{0}+\beta_{0} W, U_{3}:=U_{3}^{0}+\beta_{0}\left(h_{2}^{+}+h_{2}^{-}\right), U_{4}:=U_{4}^{0}+\beta_{0} Z_{4}$, so

$$
\left\{\zeta_{5}\left(U_{1}, U_{2}, U_{3}, U_{4}\right)\right\}=0 \text { in } \overline{H^{2}}\left(\widehat{\Gamma}, \mathfrak{s l}_{\rho_{\lambda}}\right)
$$

and there exists a cochain $U_{5}$ in $\overline{C^{1}}\left(\widehat{\Gamma}, \mathcal{G}_{+} \oplus \mathcal{G}_{-}\right)$such that

$$
\left\{\begin{aligned}
-\partial U_{5} & =\zeta_{5}\left(U_{1}, U_{2}, U_{3}, U_{4}\right) \\
j^{*} U_{5} & =U_{5}
\end{aligned}\right.
$$

Suppose that for all $1 \leq i \leq n-1$, there exists a cochain $U_{2 i+1} \in \overline{C^{1}}\left(\widehat{\Gamma}, \mathcal{G}_{+} \oplus \mathcal{G}_{-}\right)$ and a cochain $U_{2 i+2} \in \overline{C^{1}}(\widehat{\Gamma}, \mathcal{H})$ such that

$$
\left\{\begin{array} { r l } 
{ - \partial U _ { 2 i + 1 } } & { = \zeta _ { 2 i + 1 } ( U _ { 1 } , \ldots , U _ { 2 i } ) } \\
{ j ^ { * } U _ { 2 i + 1 } } & { = U _ { 2 i + 1 } }
\end{array} \quad \left\{\begin{array}{rl}
-\partial U_{2 i+2} & =\zeta_{2 i+1}\left(U_{1}, \ldots, U_{2 i+1}\right) \\
j^{*} U_{2 i+2} & =-U_{2 i+2}
\end{array}\right.\right.
$$

and consider the $2-$ cocycle $\zeta_{2 n+1}\left(U_{1}, \ldots, U_{2 n}\right) \in \overline{Z^{2}}\left(\widehat{\Gamma}, \mathcal{G}_{+} \oplus \mathcal{G}_{-}\right)$. If the latter is cohomologous to zero in $\overline{H^{2}}\left(\widehat{\Gamma}, \mathcal{G}_{+} \oplus \mathcal{G}_{-}\right)$we are done, if not, there exists $\lambda_{2 n+1} \in \mathbb{C}^{*}$ such that

$$
\left\{\zeta_{2 n+1}\left(U_{1}, \ldots, U_{2 n}\right)\right\}=\lambda_{2 n+1}\left(\left\{\zeta_{3}\left(W+V_{+}, h_{2}^{+}\right)+\zeta_{3}\left(W+V_{-}, h_{2}^{-}\right)\right\}\right) .
$$

Now for $\beta \in \mathbb{C}$,

$$
\begin{aligned}
& \left\{\zeta_{2 n+1}\left(U_{1}, \ldots, U_{2 n-2}+\beta W, U_{2 n-1}+\beta\left(h_{2}^{+}+h_{2}^{-}\right), U_{2 n}+\beta Z_{4}\right)\right\} \\
& =\left\{\zeta_{2 n+1}\left(U_{1}, \ldots, U_{2 n}\right)\right\}+\beta\left\{\eta_{4}\right\}+\beta \beta_{0}\left(\left\{\zeta_{3}\left(W+V_{+}, h_{2}^{+}\right)+\zeta_{3}\left(W+V_{-}, h_{2}^{-}\right)\right\}\right) \\
& =\left(\lambda_{2 n+1}+\beta \delta+\beta \beta_{0}\right)\left(\left\{\zeta_{3}\left(W+V_{+}, h_{2}^{+}\right)+\zeta_{3}\left(W+V_{-}, h_{2}^{-}\right)\right\}\right) .
\end{aligned}
$$

As $\beta_{0}+\delta \neq 0$ (by our assumption on $\lambda_{5}$ ), for $\beta=-\left(\lambda_{2 n+1}\right) /\left(\beta_{0}+\delta\right)$ we have

$$
\begin{aligned}
\left\{\zeta_{2 n+1}\left(U_{1}, \ldots, U_{2 n-2}+\beta W, U_{2 n-1}+\beta\left(h_{2}^{+}+h_{2}^{-}\right), U_{2 n}+\beta Z_{4}\right)\right\} & =0 \\
& \text { in } \overline{H^{2}}\left(\widehat{\Gamma}, \mathfrak{s l} \rho_{\lambda}\right) .
\end{aligned}
$$

Let then $U_{2 n+1}$ be a cochain in $\overline{C^{1}}\left(\widehat{\Gamma}, \mathcal{G}_{+} \oplus \mathcal{G}_{-}\right)$which solves $\left(E_{2 n+1}\right)$ such that $j^{*} U_{2 n+1}=U_{2 n+1}$, (such a cochain exists, cf [4] for the details).

This finishes the resolution of the infinite sequence of obstruction equations.

Now let $q: \Gamma \rightarrow \widehat{\Gamma}$ denote the natural map and $U_{t}=\sum_{i \geq 1} U_{i} t^{i}$ be the formal curve constructed above. Then by a theorem of M Artin [1] there exists a convergent curve $\tilde{U}_{t}$

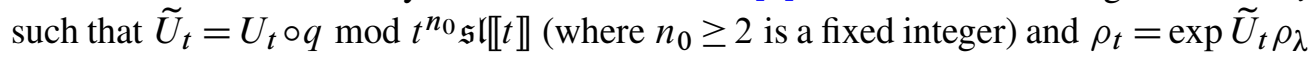
is an arc of representations of $\Gamma$ in SL for $t>0$ (see Ben Abdelghani and Lines [4] for more details). 
This proves that $U_{1}=V_{+}+V_{-} \in \mathrm{TC}_{\rho_{\lambda}}$ and, by Lemma 3.1, $Z^{1}\left(\Gamma, \mathcal{G}_{+}\right) \oplus Z^{1}\left(\Gamma, \mathcal{G}_{-}\right) \subset$ $\mathrm{TC}_{\rho_{\lambda}}$.

Now, for all $x \in \mathbb{C}^{*}$, we have

$$
\begin{aligned}
\left\{\zeta _ { 3 } \left(x W+U_{1}, x\left(h_{2}^{+}+\right.\right.\right. & \left.\left.\left.h_{2}^{-}\right)+U_{2}\right)\right\} \\
& =x^{2}\left\{\zeta_{3}\left(W+V_{+}, h_{2}^{+}\right)+\zeta_{3}\left(W+V_{-}, h_{2}^{-}\right)\right\}+\left\{\zeta_{3}\left(U_{1}, U_{2}\right)\right\} .
\end{aligned}
$$

As $r=2$, and by Proposition 4.1, $\left\{\zeta_{3}\left(W+V_{+}, h_{2}^{+}\right)+\zeta_{3}\left(W+V_{-}, h_{2}^{-}\right)\right\} \neq 0$. Moreover, $\left\{\zeta_{3}\left(U_{1}, U_{2}\right)\right\}=0$, so for all $x \in \mathbb{C}^{*}, x W+U_{1} \notin \mathrm{TC}_{\rho_{\lambda}}^{3}$. Thus

$$
\begin{array}{r}
\operatorname{TC}_{\rho_{\lambda}}=\operatorname{TC}_{\rho_{\lambda}}^{3}=Z^{1}(\Gamma, \mathcal{H}) \oplus B^{1}\left(\Gamma, \mathcal{G}_{+}\right) \oplus B^{1}\left(\Gamma, \mathcal{G}_{-}\right) \cup Z^{1}\left(\Gamma, \mathcal{G}_{+}\right) \oplus Z^{1}\left(\Gamma, \mathcal{G}_{-}\right) \\
\varsubsetneqq C Q_{\rho_{\lambda}}=Z^{1}\left(\Gamma, \mathfrak{s l}_{\rho_{\lambda}}\right) .
\end{array}
$$

This finishes the proof when $r=2$ and $\left\{\zeta_{3}\left(U_{1}, U_{2}\right)\right\}=0$.

\subsection{The case where $r \geq 2$ and $\left\{\zeta_{3}\left(U_{1}, U_{2}\right)\right\} \neq 0$ in $H^{2}\left(\Gamma, \mathfrak{s l}_{\rho_{\lambda}}\right)$}

To proceed with the proof in this case we need the following proposition which is based on a result of [16]:

Proposition 4.4 If $r \geq 2$ and $\left\{\zeta_{3}\left(U_{1}, U_{2}\right)\right\} \neq 0$ in $H^{2}\left(\Gamma, \mathfrak{s l}_{\rho_{\lambda}}\right)$ then the tangent cone $\mathrm{TC}_{\rho_{\lambda}}$ contains an irreducible component of dimension 4 .

Proof Suppose that $r \geq 2$ and $\left\{\zeta_{3}\left(U_{1}, U_{2}\right)\right\} \neq 0$ in $H^{2}\left(\Gamma, \mathfrak{s l}_{\rho_{\lambda}}\right)$, then we obtain $\operatorname{dim}_{\mathbb{C}} Z^{1}\left(\Gamma, \mathfrak{s l}_{\phi_{\lambda}}\right)=4$ (see Lemma 4.3). As $\phi_{\lambda}$ is nonabelian, $\operatorname{dim}_{\mathbb{C}} H^{1}\left(\Gamma, \mathfrak{s l}_{\phi_{\lambda}}\right)=1$. By [16, Lemma 7.5], every cocycle in $Z^{1}\left(\Gamma, \mathfrak{s l}_{\phi_{\lambda}}\right)$ is integrable. So the representation $\phi_{\lambda}$ is a smooth point of $R(\Gamma)$ with local dimension 4 . As $\rho_{\lambda}$ is in the adherence of the orbit of $\phi_{\lambda}$, it is contained in the same irreducible component $R_{\lambda}(\Gamma)$ of dimension 4. Thus, we conclude the tangent cone of $R(\Gamma)$ at $\rho_{\lambda}$ contains an irreducible component of dimension 4.

Now if $r=2$ and $\left\{\zeta_{3}\left(U_{1}, U_{2}\right)\right\} \neq 0$ in $H^{2}\left(\Gamma, \mathfrak{s l}_{\rho_{\lambda}}\right)$, there exists $\beta \in \mathbb{C}^{*}$ such that

$$
\left\{\zeta_{3}\left(U_{1}, U_{2}\right)\right\}=\beta\left\{\zeta_{3}\left(W+V_{+}, h_{2}^{+}\right)+\zeta_{3}\left(W+V_{-}, h_{2}^{-}\right)\right\},
$$

and for $\beta_{0}= \pm \sqrt{-\beta}$, we obtain

$$
\left\{\zeta_{3}\left(\beta_{0} W+U_{1}, U_{2}+\beta_{0}\left(h_{2}^{+}+h_{2}^{-}\right)\right)\right\}=0 \text { in } H^{2}\left(\Gamma, \mathfrak{s l}_{\rho_{\lambda}}\right) .
$$


Using Lemma 3.1, we deduce that the cubic tangent cone is given by

$$
\begin{array}{r}
\operatorname{TC}_{\rho_{\lambda}}^{3}(\Gamma)=\left\{\delta\left(\beta_{0} W+x V_{+}+x^{-1} V_{-}\right)+B_{+}+B_{-} \mid \delta \in \mathbb{C}, x \in \mathbb{C}^{*}, B_{ \pm} \in B^{1}\left(\Gamma, \mathcal{G}_{ \pm}\right)\right\} \\
\cup\left\{\delta W+B_{+}+B_{-} \mid \delta \in \mathbb{C}, B_{ \pm} \in B^{1}\left(\Gamma, \mathcal{G}_{ \pm}\right)\right\} .
\end{array}
$$

Hence, it has two components: one of dimension 4 and one of dimension 3 ; so it is of dimension 4. As $\mathrm{TC}_{\rho_{\lambda}} \subset \mathrm{TC}_{\rho_{\lambda}}^{3}$ and $\mathrm{TC}_{\rho_{\lambda}}$ has an irreducible component of dimension 4 , we conclude that $\mathrm{TC}_{\rho_{\lambda}}=\mathrm{TC}_{\rho_{\lambda}}^{3}$ and $R(\Gamma)$ is of dimension 4 locally at $\rho_{\lambda}$.

Now, for the second part of the Theorem 1.1, we use an argument by contradiction. Suppose that $r \geq 3$ and $\left\{\zeta_{3}\left(U_{1}, U_{2}\right)\right\} \neq 0$ in $H^{2}\left(\Gamma, \mathfrak{s l}_{\rho_{\lambda}}\right)$. Then, by Proposition 4.1, $\left\{\zeta_{3}\left(W+V_{ \pm}, h_{2}^{ \pm}\right)\right\}=0$ and for all $\delta \in \mathbb{C},\left\{\zeta_{3}\left(\delta W+U_{1}, U_{2}+\delta\left(h_{2}^{+}+h_{2}^{-}\right)\right)\right\} \neq 0$ in $H^{2}\left(\Gamma, \mathfrak{s l}_{\rho_{\lambda}}\right)$. So, we will have

$$
\mathrm{TC}_{\rho_{\lambda}}^{3}=Z^{1}(\Gamma, \mathcal{H}) \oplus B^{1}\left(\Gamma, \mathcal{G}_{+}\right) \oplus B^{1}\left(\Gamma, \mathcal{G}_{-}\right),
$$

and $\mathrm{TC}_{\rho_{\lambda}}=\mathrm{TC}_{\rho_{\lambda}}^{3}$ will be of dimension 3. This is impossible by Proposition 4.4.

Thus, we conclude that if $r \geq 3$ then necessarily $\left\{\zeta_{3}\left(U_{1}, U_{2}\right)\right\}=0$ in $H^{2}\left(\Gamma, \mathfrak{s l}_{\rho_{\lambda}}\right)$ and for all $\delta \in \mathbb{C}, \delta W+U_{1} \in \mathrm{TC}_{\rho_{\lambda}}^{3}$. This implies that $\operatorname{TC}_{\rho_{\lambda}}^{3}=Z^{1}\left(\Gamma, \mathfrak{s l}_{\rho_{\lambda}}\right)$ is of dimension 5 and, in particular, $\mathrm{TC}_{\rho_{\lambda}} \varsubsetneqq \mathrm{TC}_{\rho_{\lambda}}^{3}$ because there are no 5-dimensional components going through $\rho_{\lambda}$.

\section{Applications and examples}

As an application to Theorem 1.1, we prove Proposition 1.2 which describes the local geometry of the representation variety at an abelian representation and at a reducible nonabelian representation with the same character $\chi_{\lambda}$ as the abelian one. Further, we prove Theorem 1.3, that describes the local geometry of the character variety at $\chi_{\lambda}$ and we illustrate our discussion with the examples of the two-bridge knot $b(49,17)$ and the knot $8_{20}$.

Let $\alpha:=\lambda^{2}$ be a root of $\Delta_{1}$ and suppose that the $(t-\alpha)-$ torsion of the Alexander module $H_{1}\left(\pi_{1}(\widetilde{M}), \mathbb{C}\right)$ is cyclic of the form $\Lambda /(t-\alpha)^{2} \oplus \Lambda /\left(t-\alpha^{-1}\right)^{2}, r \geq 2$. Let $\rho_{\lambda}: \Gamma \rightarrow$ SL be the abelian representation given by $\rho_{\lambda}(\mu)=\operatorname{diag}\left(\lambda, \lambda^{-1}\right)$ and $\phi_{\lambda}: \Gamma \rightarrow$ SL be the reducible nonabelian representation given by $\phi_{\lambda}=\exp \left(V_{+}\right) \rho_{\lambda}$.

Proof of Proposition 1.2 (1) Suppose that $\operatorname{dim}_{\mathbb{C}} Z^{1}\left(\Gamma, \mathfrak{s l}_{\phi_{\lambda}}\right)=4$, then by Section $4.4, \phi_{\lambda}$ is a smooth point of $R(\Gamma)$ which is contained in a unique 4-dimensional component $R_{\lambda}(\Gamma)$. In particular, $\phi_{\lambda}$ is scheme reduced and $\mathrm{TC}_{\phi_{\lambda}}=Z^{1}\left(\Gamma, \mathfrak{s l}_{\phi_{\lambda}}\right)=$ $\left\langle\left(W+h_{2}^{+}-1 / 2\left[W, V_{+}\right]\right)\right\rangle \oplus B^{1}\left(\Gamma, \mathfrak{s l}_{\Phi_{\lambda}}\right)$. 
Now to prove the fact that $S(\Gamma)$ and $R_{\lambda}(\Gamma)$ are the only irreducible components of $R(\Gamma)$ that contain $\rho_{\lambda}$ and that $\rho_{\lambda}$ is a smooth point of both varieties, it suffices to proceed exactly as in $[4$, Corollary $5.3 ; 17$, Corollary 1.3$]$.

(2) If $\operatorname{dim}_{\mathbb{C}} Z^{1}\left(\Gamma, \mathfrak{s l}_{\phi_{\lambda}}\right)=5$, then by Section 4.3, $\rho_{\lambda}$ is contained in at least one 4-dimensional component which contains irreducible representations. By [4, Theorem 5.2, ii)], the nonabelian representation $\phi_{\lambda}$ is contained in each 4-dimensional component of $R(\Gamma)$ containing $\rho_{\lambda}$ and containing irreducible representations.

By the analysis of the tangent cone in Section 4.3, we proved the existence of an arc of irreducible representations with endpoint $\rho_{\lambda}$ of the form

$$
\rho_{t}=\exp \left(t U_{1}+t^{2}\left(U_{2}^{0}+\beta_{0} W\right)+t^{3}\left(U_{3}^{0}+\beta_{0}\left(h_{2}^{+}+h_{2}^{-}\right)\right)+o\left(t^{4}\right)\right) \rho_{\lambda} .
$$

Set, for $t>0, \Phi_{t}=A_{t}^{-1} \rho_{t} A_{t}$ with

$$
A_{t}=\left(\begin{array}{cc}
t^{1 / 2} & 0 \\
0 & t^{-1 / 2}
\end{array}\right)
$$

Then

$$
\Phi_{t}=\exp \left(V_{+}+t^{2}\left(V_{-}+U_{2}^{0}+\beta_{0} W+\left(U_{3}^{0}\right)^{+}+\beta_{0} h_{2}^{+}\right)+o\left(t^{3}\right)\right) \rho_{\lambda},
$$

and $\Phi_{t}$ is an arc of irreducible representations with endpoint $\phi_{\lambda}$. Using the CampbellHausdorff formula [6, Chapter II, Section 6.5], one can see that $\Phi_{t}$ is tangent to the cocycle

$$
\begin{aligned}
Z=\beta_{0}\left(W+h_{2}^{+}-\frac{1}{2}\left[W, V_{+}\right]\right)+V_{-}+ & U_{2}^{0}+\frac{1}{2}\left[V_{+}, V_{-}\right] \\
& +\left(U_{3}^{0}\right)^{+}+\frac{1}{2}\left[V_{+}, U_{2}^{0}\right]-\frac{1}{6}\left[V_{+},\left[V_{+}, V_{-}\right]\right] .
\end{aligned}
$$

This proves that $Z \in \mathrm{TC}_{\phi_{\lambda}}$. As we have two possible choices for $\beta_{0}$, which will be denoted $\beta_{1}, \beta_{2}$, we have two possible choices for the cocycle $Z$ which we denote by $Z_{1}$ and $Z_{2}$.

As $\beta_{0} \neq 0$ and by Lemma $4.3,\left\langle Z_{1}, Z_{2}\right\rangle \oplus B^{1}\left(\Gamma, \mathfrak{s l}_{\phi_{\lambda}}\right)=Z^{1}\left(\Gamma, \mathfrak{s l}_{\phi_{\lambda}}\right)$ and $Z_{1}, Z_{2} \in$ $\mathrm{TC}_{\phi_{\lambda}} \subset T_{\phi_{\lambda}}^{\mathrm{Zar}} R(\Gamma)$, so $Z^{1}\left(\Gamma, \mathfrak{s l}_{\phi_{\lambda}}\right) \subset T_{\phi_{\lambda}}^{\mathrm{Zar}} R(\Gamma)$ and $Z^{1}\left(\Gamma, \mathfrak{s l}_{\phi_{\lambda}}\right)=T_{\phi_{\lambda}}^{\mathrm{Zar}} R(\Gamma)$. This proves that $\phi_{\lambda}$ is scheme reduced.

On the other hand, it is easy to see that there is no 5-component containing $\phi_{\lambda}$ because there is no 5-component containing $\rho_{\lambda}$. This implies that $\operatorname{dim}_{\mathbb{C}} \mathrm{TC}_{\phi_{\lambda}}=4$ and has at least two components:

$$
\left\langle Z_{1}\right\rangle \oplus B^{1}\left(\Gamma, \mathfrak{s l}_{\phi_{\lambda}}\right) \cup\left\langle Z_{2}\right\rangle \oplus B^{1}\left(\Gamma, \mathfrak{s l}_{\phi_{\lambda}}\right) \subset \mathrm{TC}_{\phi_{\lambda}} .
$$


It remains to prove that $\mathrm{QC}_{\phi_{\lambda}} \varsubsetneqq Z^{1}\left(\Gamma, \mathfrak{s l}_{\phi_{\lambda}}\right)$ and that

$$
\mathrm{TC}_{\phi_{\lambda}}=\mathrm{QC}_{\phi_{\lambda}}=\left\langle Z_{1}\right\rangle \oplus B^{1}\left(\Gamma, \mathfrak{s l}_{\phi_{\lambda}}\right) \cup\left\langle Z_{2}\right\rangle \oplus B^{1}\left(\Gamma, \mathfrak{s l}_{\phi_{\lambda}}\right) .
$$

Let $\mathcal{U}=\alpha_{1} Z_{1}+\alpha_{2} Z_{2} \in \mathrm{QC}_{\phi_{\lambda}}$. Our goal is to prove that either $\alpha_{1}=0$ or $\alpha_{2}=0$. Let $\phi_{t}$ be the formal deformation modulo order 2 of $\phi_{\lambda}$ given by

$$
\phi_{t}=\exp \left(t \mathcal{U}+o\left(t^{2}\right)\right) \exp \left(V_{+}\right) \rho_{\lambda} .
$$

Set $\rho_{t}=B_{t} \phi_{t} B_{t}^{-1}$ with

Then

$$
B_{t}=\left(\begin{array}{cc}
t^{1 / 4} & 0 \\
0 & t^{-1 / 4}
\end{array}\right)
$$

$$
\begin{aligned}
\rho_{t}= & B_{t} \exp \left(t\left(\alpha_{1} Z_{1}+\alpha_{2} Z_{2}\right)+o\left(t^{2}\right)\right) \exp \left(V_{+}\right) B_{t}^{-1} \rho_{\lambda} \\
= & \exp \left(t\left(\alpha_{1}\left(Z_{1}^{0}+t^{1 / 2} Z_{1}^{+}+t^{-1 / 2} Z_{1}^{-}\right)+\alpha_{2}\left(Z_{2}^{0}+t^{1 / 2} Z_{2}^{+}+t^{-1 / 2} Z_{2}^{-}\right)+o\left(t^{2}\right)\right)\right. \\
& \quad \times \exp \left(t^{1 / 2} V_{+}\right) \rho_{\lambda} \\
= & \exp \left(t^{1 / 2}\left(\alpha_{1} Z_{1}^{-}+\alpha_{2} Z_{2}^{-}\right)+t\left(\alpha_{1} Z_{1}^{0}+\alpha_{2} Z_{2}^{0}\right)+o\left(t^{3 / 2}\right)\right) \exp \left(t^{1 / 2} V_{+}\right) \rho_{\lambda} \\
= & \exp \left(t^{1 / 2}\left(V_{+}+\alpha_{1} Z_{1}^{-}+\alpha_{2} Z_{2}^{-}\right)+t\left(\alpha_{1} Z_{1}^{0}+\alpha_{2} Z_{2}^{0}+\frac{1}{2}\left[\alpha_{1} Z_{1}^{-}+\alpha_{2} Z_{2}^{-}, V_{+}\right]\right)\right. \\
& \left.\quad+o\left(t^{3 / 2}\right)\right) \rho_{\lambda} .
\end{aligned}
$$

As

$$
\left\{\begin{aligned}
V_{+}+\alpha_{1} Z_{1}^{-}+\alpha_{2} Z_{2}^{-} & =V_{+}+\left(\alpha_{1}+\alpha_{2}\right) V_{-} \\
\alpha_{1} Z_{1}^{0}+\alpha_{2} Z_{2}^{0}+\frac{1}{2}\left[\alpha_{1} Z_{1}^{-}+\alpha_{2} Z_{2}^{-}, V_{+}\right] & =\left(\alpha_{1} \beta_{1}+\alpha_{2} \beta_{2}\right) W+\left(\alpha_{1}+\alpha_{2}\right) U_{2}^{0},
\end{aligned}\right.
$$

we obtain

$$
\rho_{t}=\exp \left(t^{1 / 2}\left(V_{+}+\left(\alpha_{1}+\alpha_{2}\right) V_{-}\right)+t\left(\left(\alpha_{1} \beta_{1}+\alpha_{2} \beta_{2}\right) W+\left(\alpha_{1}+\alpha_{2}\right) U_{2}^{0}\right)+o\left(t^{3 / 2}\right)\right) \rho_{\lambda} \text {. }
$$

The analysis of the tangent cone at $\rho_{\lambda}$ in Section 4.3 shows that we have necessarily

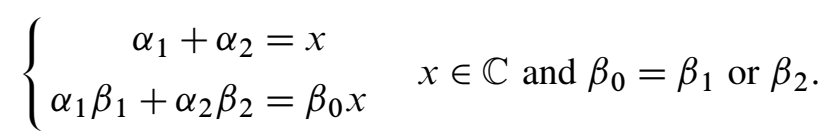

If $\beta_{0}=\beta_{1}$ we obtain $x=\alpha_{1}$ and if $\beta_{0}=\beta_{2}$ we obtain $x=\alpha_{2}$. Thus $\alpha_{2}=0$ or $\alpha_{1}=0$. This implies that $\mathcal{U}=x Z_{1}$ or $\mathcal{U}=x Z_{2} ; x \in \mathbb{C}$. So we conclude that

$$
\mathrm{QC}_{\phi_{\lambda}}=\left\langle Z_{1}\right\rangle \oplus B^{1}\left(\Gamma, \mathfrak{s l}_{\phi_{\lambda}}\right) \cup\left\langle Z_{2}\right\rangle \oplus B^{1}\left(\Gamma, \mathfrak{s l}_{\phi_{\lambda}}\right) \subset \mathrm{TC}_{\phi_{\lambda}} .
$$

As $\mathrm{TC}_{\phi_{\lambda}} \subset \mathrm{QC}_{\phi_{\lambda}}$, we are done. 
Remarks (1) If $r=2$ and $\operatorname{dim}_{\mathbb{C}} Z^{1}\left(\Gamma, \mathfrak{s l}_{\phi_{\lambda}}\right)=5$, then $\phi_{\lambda}$ is a quadratic singularity of $R(\Gamma)$.

(2) If $r \geq 3$ then $\operatorname{dim}_{\mathbb{C}} Z^{1}\left(\Gamma, \mathfrak{s l}_{\phi_{\lambda}}\right)=5$ and $\phi_{\lambda}$ is necessarily a singular point of $R(\Gamma)$.

Proof of Theorem 1.3 (1) By [17, Lemma 5.4], the projection $\pi: R(\Gamma) \rightarrow X(\Gamma)$ is open at the abelian representation $\rho_{\lambda}$; ie if $U$ is a classical neighborhood of $\rho_{\lambda}$ then $\pi(U)$ is a classical neighborhood of $\chi_{\lambda}$. Moreover, by Proposition 1.2, the analytic germ of $R(\Gamma)$ at $\rho_{\lambda}$ has only two irreducible components which are precisely the germ of $R_{\lambda}(\Gamma)$ and the germ of $S(\Gamma)$. These two results imply that $X_{\lambda}(\Gamma)$ and $Y(\Gamma)$ are the unique irreducible components of $X(\Gamma)$ that contain $\chi_{\lambda}$.

Now, Proposition 5.6 of [17] asserts that $\chi_{\lambda}$ is a smooth point of the curve $Y(\Gamma)$. So it remains to prove that $\chi_{\lambda}$ is a smooth point of the curve $X_{\lambda}(\Gamma)$ and that $X_{\lambda}(\Gamma)$ and $Y(\Gamma)$ are tangent curves. As the set of irreducible representations of $R_{\lambda}(\Gamma)$ is dense in $R_{\lambda}(\Gamma), X_{\lambda}(\Gamma) \subset \overline{X^{\text {irr }}(\Gamma)}$. By Theorem A of [7], $\chi_{\lambda}$ is a smooth point of $\overline{X^{\text {irr }}(\Gamma)}$ and therefore of the irreducible component $X_{\lambda}(\Gamma)$. By [11, Corollary 1.5.3], $\operatorname{dim} X_{\lambda}(\Gamma)=\operatorname{dim} R_{\lambda}(\Gamma)-3=1$ and $X_{\lambda}(\Gamma)$ is a curve in $X(\Gamma)$.

We recall that the function algebra $\mathbb{C}[X(\Gamma)]$ is finitely generated by the evaluation functions $I_{\gamma}: X(\Gamma) \rightarrow \mathbb{C} ; \chi_{\rho} \mapsto I_{\gamma}\left(\chi_{\rho}\right)=\operatorname{tr}(\rho(\gamma))$, where $\gamma \in \Gamma$, which are regular maps [11]. Let $W \in Z^{1}(\Gamma, \mathbb{C})$ be an abelian cocycle such that

$$
W\left(S_{1}\right)=\left(\begin{array}{cc}
1 & 0 \\
0 & -1
\end{array}\right) .
$$

Then $\rho_{s}(\gamma)=\exp (s W(\gamma)) \rho_{\lambda}(\gamma), \gamma \in \Gamma$, is a path of abelian representations with endpoint $\rho_{\lambda}$ and tangent to $W$ at $s=0$. For $\gamma \in \Gamma$, we write

$$
W(\gamma)=\left(\begin{array}{cc}
|\gamma| & 0 \\
0 & -|\gamma|
\end{array}\right)
$$

with $|\gamma|=\Phi(\gamma)$ where $\Phi: \Gamma \rightarrow \Gamma / \Gamma^{\prime} \cong \mathbb{Z}$ designs the canonical projection. Since, for $\gamma \in \Gamma$,

$$
I_{\gamma}\left(\pi\left(\rho_{s}\right)\right)=\operatorname{tr} \rho_{s}(\gamma)=\lambda^{|\gamma|}+\frac{1}{\lambda^{|\gamma|}}+s|\gamma|\left(\lambda^{|\gamma|}-\frac{1}{\lambda^{|\gamma|}}\right)+o\left(s^{2}\right),
$$

it follows that $d_{\chi_{\lambda}} I_{S_{i}}\left(d_{\rho_{\lambda}} \pi(W)\right)=\lambda-1 / \lambda \neq 0$. Thus, $d_{\rho_{\lambda}} \pi(W)$ is a basis for $T_{\chi_{\lambda}}^{\mathrm{Zar}} Y(\Gamma) \cong \mathbb{C}$.

On the other hand, we know by the proof of Proposition 1.2 that

$$
\phi_{s}(\gamma)=\exp \left(s\left(W(\gamma)+h_{2}^{+}(\gamma)-1 / 2\left[W(\gamma), V_{+}(\gamma)\right]\right)+o\left(s^{2}\right)\right) \phi_{\lambda}(\gamma)
$$


is a path of irreducible representations in $R_{\lambda}(\Gamma)$ with endpoint $\phi_{\lambda}$ and tangent to $\mathcal{U}=W+h_{2}^{+}-1 / 2\left[W, V_{+}\right]$at $s=0$. As adding elements of $\mathcal{G}_{+}$to a deformation of $\rho_{\lambda}$ or $\phi_{\lambda}$ do not change the trace at the first order, we obtain for $\gamma \in \Gamma$

$$
I_{\gamma}\left(\pi\left(\phi_{s}\right)\right)=\operatorname{tr} \phi_{s}(\gamma)=\lambda^{|\gamma|}+\frac{1}{\lambda^{|\gamma|}}+s|\gamma|\left(\lambda^{|\gamma|}-\frac{1}{\lambda^{|\gamma|}}\right)+o\left(s^{2}\right) .
$$

It follows that for all $\gamma \in \Gamma$

$$
d_{\chi_{\lambda}} I_{\gamma}\left(d_{\phi_{\lambda}} \pi(\mathcal{U})\right)=|\gamma|\left(\lambda^{|\gamma|}-\frac{1}{\lambda^{|\gamma|}}\right)=d_{\chi_{\lambda}} I_{\gamma}\left(d_{\rho_{\lambda}} \pi(W)\right) .
$$

Therefore $d_{\phi_{\lambda}} \pi(\mathcal{U})$ is a basis of $T_{\chi_{\lambda}}^{\mathrm{Zar}} X_{\lambda}(\Gamma) \cong \mathbb{C}$ and $d_{\phi_{\lambda}} \pi(\mathcal{U})=d_{\rho_{\lambda}} \pi(W)$. Thus, $T_{\chi_{\lambda}}^{\mathrm{Zar}} Y(\Gamma)=T_{\chi_{\lambda}}^{\mathrm{Zar}} X_{\lambda}(\Gamma)$ and $Y(\Gamma)$ and $X_{\lambda}(\Gamma)$ are tangent curves.

The last assertion is a consequence of [7, Theorem A].

(2) By the second assertion of Proposition 1.2, the representation $\phi_{\lambda}$ is a singular point of $R(\Gamma)$ which is contained in at least two 4-dimensional local components of $R(\Gamma)$ which contain irreducible representations. Thus, as $\rho_{\lambda}$ is contained in the adherence of the orbit of $\phi_{\lambda}$, the abelian representation $\rho_{\lambda}$ is itself contained in these 4-dimensional components of $\overline{R^{\text {irr }}(\Gamma)}$. Using again the fact that the projection $\pi: R(\Gamma) \rightarrow X(\Gamma)$ is open at the abelian representation $\rho_{\lambda}$, we can assert that $\chi_{\lambda}$ is contained in at least 2 irreducible components of $\overline{X^{\text {irr }}(\Gamma)}$. This implies, in particular, that $\chi_{\lambda}$ is not a smooth point of $\overline{X^{\operatorname{irr}}(\Gamma)}$.

\subsection{The knot $b(49,17)$}

Let $K \subset S^{3}$ be the 2-bridge knot $b(49,17)$ [10]. This is an alternating knot with 12 crossings in a minimal projection and with Alexander polynomial $\Delta_{1}(t)=\left(2 t^{2}-3 t+2\right)^{2}$. Let $\lambda$ be a complex number on the unit circle such that $\Delta_{1}\left(\lambda^{2}\right)=0$. Then $\alpha=\lambda^{2}$ is a double root of the Alexander polynomial and the Alexander module with complex coefficients of $b(49,17)$ is given by $\Lambda /(t-\alpha)^{2} \oplus \Lambda /\left(t-\alpha^{-1}\right)^{2}[10]$.

A computer supported calculation [17] shows that $\operatorname{dim}_{\mathbb{C}} Z^{1}\left(\Gamma, \mathfrak{s l}_{\phi_{\lambda}}\right)=4$. Section 4.4 shows that the 2 -bridge knot $b(49,17)$ admits a strongly nonquadratic singularity in the SL representation variety. Moreover, by Theorem 1.3, in this case every reducible but nonabelian representation $\phi_{\lambda}$ such that $\chi_{\phi_{\lambda}}=\chi_{\lambda}$ is a smooth point of the representation variety and the components $\left.X_{\lambda}(\Gamma)\right)$ and $Y(\Gamma)$ are tangent at $\chi_{\lambda}$.

\subsection{The knot $8_{20}$}

Let $K$ be the knot $8_{20}$ in Rolfsen's list. We have that $\Delta_{1}(t)=\left(t^{2}-t+1\right)^{2}$ and we denote $\lambda:=\exp (i \pi / 6)$. Then this knot has an Alexander module with 
complex coefficients of the form $\Lambda /(t-\alpha)^{2} \oplus \Lambda /\left(t-\alpha^{-1}\right)^{2}$ [10]. But this time $\operatorname{dim}_{\mathbb{C}} Z^{1}\left(\Gamma, \mathfrak{s l}_{\phi_{\lambda}}\right)=5$. So by Section 4.3 , we have again a strongly nonquadratic singularity. Moreover, by Theorem 1.3, the character $\chi_{\lambda}$ is not a smooth point of the character variety of irreducible representations. More precisely, there are at least two irreducible components of $\overline{X^{\operatorname{irr}}(\Gamma)}$ passing through $X_{\lambda}$.

Remarks (1) The examples of the two-bridge knot $b(49,17)$ and the knot $8_{20}$ were treated in [17], but our methods differ from theirs.

(2) Each one of the knots $b(49,17)$ and $8_{20}$ has a cyclic Alexander module of order 2 . But our study of the local structure of their representation and character varieties proves that one cannot decide of this local structure unless one calculates the dimension of the space of cocycles $Z^{1}\left(\Gamma, \mathfrak{s l}_{\phi_{\lambda}}\right)$. So this remains an open question: can we guess the local structure without cohomological computations?

\section{Proof of Proposition 4.1}

We will construct inductively the cochains $h_{i}^{+}: \Gamma \rightarrow \mathcal{G}_{+}, 2 \leq i \leq r$, such that the cochain $\zeta_{j}\left(W+V_{+}, h_{2}^{+}, \ldots, h_{j-1}^{+}\right)$is defined for every $3 \leq j \leq r$ and the class $\left\{\zeta_{r+1}\left(W+V_{+}, h_{2}^{+}, \ldots, h_{r}^{+}\right)\right\} \neq 0$ in $H^{2}\left(\Gamma, \mathcal{G}_{+}\right)$.

Consider the second obstruction equation

$$
-\partial h_{2}^{+}=\zeta_{2}\left(W+V_{+}\right)=\frac{1}{2} W \smile V_{+}+\frac{1}{2} V_{+} \smile W .
$$

Observe first that we may suppose $h_{2}^{+}(\mu)=0$. Indeed, if $h_{2}^{+}(\mu)=g \in \mathcal{G}_{+}$, we can replace $h_{2}^{+}$by $h_{2}^{+}+\partial C$ where $C$ is the $0-$ cochain with value $(1 /(1-\alpha)) g$. Then using $\left(E_{2}\right)$ and induction on $k$, we see that $h_{2}^{+}\left(\mu^{k}\right)=0$.

Further, for all $\gamma_{1}, \gamma_{2} \in N, h_{2}^{+}\left(\gamma_{1} \gamma_{2}\right)=h_{2}^{+}\left(\gamma_{1}\right)+h_{2}^{+}\left(\gamma_{2}\right)$. So we deduce that $\left.h_{2}^{+}\right|_{N_{2}}=$ 0 and $h_{2}^{+}$factors through $N / N_{2}$.

Now, for all $\left(x, t^{k}\right),\left(y, t^{l}\right) \in N / N_{2} \rtimes T$, Equation $\left(E_{2}\right)$ is equivalent to

$$
h_{2}^{+}\left(x+t^{k} y, t^{k+l}\right)=h_{2}^{+}\left(x, t^{k}\right)+\alpha^{k} h_{2}^{+}\left(y, t^{l}\right)+\zeta_{2}\left(W+V_{+}\right)\left(\left(x, t^{k}\right),\left(y, t^{l}\right)\right) \text {. }
$$

As $\left(x, t^{k}\right)=(x, 1)\left(0, t^{k}\right)$, we obtain the equivalent system

$$
\left\{\begin{aligned}
h_{2}^{+}(x+y, 1) & =h_{2}^{+}(x, 1)+h_{2}^{+}(y, 1) \\
h_{2}^{+}\left(x, t^{k}\right) & =h_{2}^{+}(x, 1)+\zeta_{2}\left(W+V_{+}\right)\left((x, 1),\left(0, t^{k}\right)\right) \\
h_{2}^{+}\left(t^{k} y, 1\right)-\alpha^{k} h_{2}^{+}(y, 1)=-\zeta_{2}\left(W+V_{+}\right)\left(\left(t^{k} y, 1\right),\left(0, t^{k}\right)\right) & +\zeta_{2}\left(W+V_{+}\right)\left(\left(0, t^{k}\right),(y, 1)\right) .
\end{aligned}\right.
$$


The first equation of the above system and the fact that $h_{2}^{+}$factors through $N / N_{2}$ imply that we can extend the map $N / N_{2} \rightarrow \mathcal{G}_{+} ; x \mapsto h_{2}^{+}(x, 1)$ to a $\mathbb{C}$-linear map denoted by $\varphi_{2}^{+}$. So the solution of $\left(E_{2}\right)$ is equivalent to the existence of a $\mathbb{C}$-linear $\operatorname{map} \varphi_{2}^{+}: N / N_{2} \otimes \mathbb{C} \rightarrow \mathcal{G}_{+}$such that for all $y \in N / N_{2} \otimes \mathbb{C}$ and $k \in \mathbb{Z}$ :

$$
\begin{aligned}
& \varphi_{2}^{+}\left(\left(t^{k}-\alpha^{k}\right) y\right) \\
& \quad=-\zeta_{2}\left(W+V_{+}\right)\left(\left(t^{k} y, 1\right),\left(0, t^{k}\right)\right)+\zeta_{2}\left(W+V_{+}\right)\left(\left(0, t^{k}\right),(y, 1)\right) .
\end{aligned}
$$

On the other hand, let $\left(e_{0}=[1], e_{1}=[t-\alpha], \ldots, e_{r-1}=\left[(t-\alpha)^{r-1}\right]\right)$ be a $\mathbb{C}$-basis for $\Lambda /(t-\alpha)^{r}$, then for all $0 \leq i \leq r-1$ :

$$
\left(t^{k}-\alpha^{k}\right) e_{i}=a_{1} e_{i+1}+\cdots+a_{r-1-i} e_{r-1} \quad \text { with } a_{1}=k \alpha^{k-1} .
$$

In particular

$$
\left\{\begin{aligned}
\left(t^{k}-\alpha^{k}\right) e_{r-2} & =a_{1} e_{r-1} \\
\left(t^{k}-\alpha^{k}\right) e_{r-3} & =a_{1} e_{r-2}+a_{2} e_{r-1} \\
& \vdots \\
\left(t^{k}-\alpha^{k}\right) e_{i+1} & =a_{1} e_{i+2}+\cdots+a_{r-i-2} e_{r-1} \\
\left(t^{k}-\alpha^{k}\right) e_{i} & =a_{1} e_{i+1}+\cdots+a_{r-i-1} e_{r-1} \\
\left(t^{k}-\alpha^{k}\right) e_{i-1} & =a_{1} e_{i}+\cdots+a_{r-i} e_{r-1} \\
& \vdots \\
\left(t^{k}-\alpha^{k}\right) e_{0} & =a_{1} e_{1}+\cdots+a_{r-1} e_{r-1} .
\end{aligned}\right.
$$

Letting $y=e_{i}, 0 \leq i \leq r-2$ in Equation $\left(\widetilde{E}_{2}\right)$ and using the fact that $V_{+}\left(e_{i}\right)=0$ for all $1 \leq i \leq r-1$, one can see that $\left(\widetilde{E}_{2}\right)$ is equivalent to the linear system with complex coefficients

$$
\left(\begin{array}{cccc}
0 & \cdots & 0 & a_{1} \\
0 & \cdots & a_{1} & a_{2} \\
\vdots & \ddots & \ddots & \vdots \\
\vdots & \ddots & \ddots & \vdots \\
a_{1} & a_{2} & \cdots & a_{r-1}
\end{array}\right)\left(\begin{array}{c}
\varphi_{2}^{+}\left(e_{1}\right) \\
\vdots \\
\vdots \\
\vdots \\
\varphi_{2}^{+}\left(e_{r-1}\right)
\end{array}\right)=\left(\begin{array}{c}
0 \\
\vdots \\
\vdots \\
\vdots \\
2 k \alpha^{k} W(\mu) V_{+}\left(e_{0}\right)
\end{array}\right) .
$$

As $a_{1} \neq 0$, this system has a unique solution defined by

$$
\left\{\begin{array}{l}
\varphi_{2}^{+}\left(e_{1}\right)=2 \alpha W(\mu) V_{+}\left(e_{0}\right) \\
\varphi_{2}^{+}\left(e_{i}\right)=0 \text { for all } 2 \leq i \leq r-1 .
\end{array}\right.
$$


Note that this solution does not depend on $k$, as required.

Proceeding in the same manner, we can construct for all $2 \leq j \leq r$ cochains $h_{j}^{+}: \Gamma \rightarrow \mathcal{G}_{+}$ resolving

$$
-\partial h_{j}^{+}=\zeta_{j}\left(W+V_{+}, h_{2}^{+}, \ldots, h_{j-1}^{+}\right)
$$

and such that $h_{j}^{+}(\mu)=0$, by constructing $\mathbb{C}$-linear maps $\varphi_{j}^{+}: N / N_{2} \otimes \mathbb{C} \rightarrow \mathcal{G}_{+}$ which satisfy the condition that $\left(\varphi_{j}^{+}\left(e_{1}\right), \ldots, \varphi_{j}^{+}\left(e_{r-1)}\right)\right.$ is a solution of the system

$$
\left(\begin{array}{cccc}
0 & \cdots & 0 & a_{1} \\
0 & \cdots & a_{1} & a_{2} \\
\vdots & \ddots & \ddots & \vdots \\
\vdots & \ddots & \ddots & \vdots \\
a_{1} & a_{2} & \cdots & a_{r-1}
\end{array}\right)\left(\begin{array}{c}
\varphi_{j}^{+}\left(e_{1}\right) \\
\vdots \\
\vdots \\
\vdots \\
\varphi_{j}^{+}\left(e_{r-1}\right)
\end{array}\right)=\left(\begin{array}{c}
b_{1}^{+} \\
\vdots \\
\vdots \\
\vdots \\
b_{r-1}^{+}
\end{array}\right)
$$

and $b_{i}^{+}=-\zeta_{j}\left(W+V_{+}, \varphi_{2}^{+}, \ldots, \varphi_{j-1}^{+}\right)\left(\left(t^{k} e_{i}, 1\right),\left(0, t^{k}\right)\right)$

$$
+\zeta_{j}\left(W+V_{+}, \varphi_{2}^{+}, \ldots, \varphi_{j-1}^{+}\right)\left(\left(0, t^{k}\right),\left(e_{i}, 1\right)\right)
$$

for all $1 \leq i \leq r$.

It is easy to see that for all $2 \leq j \leq r$,

$$
\left\{\begin{array}{c}
\varphi_{j}^{+}\left(e_{j}\right)=\cdots=\varphi_{j}^{+}\left(e_{r-1}\right)=0 \\
\varphi_{j}^{+}\left(e_{j-1}\right)=(2 \alpha W(\mu))^{j-1} V_{+}\left(e_{0}\right)
\end{array}\right.
$$

and that for all $2 \leq j \leq r$ and for all $\left(x, t^{k}\right) \in \Gamma=N \rtimes T$,

$$
h_{j}^{+}\left(x, t^{k}\right)=\varphi_{j}^{+}(x)+\zeta_{j}\left(W+V_{+}, h_{2}^{+}, \ldots, h_{j-1}^{+}\right)\left((x, 1),\left(0, t^{k}\right)\right) .
$$

Now to prove that $\left\{\zeta_{r+1}\left(W+V_{+}, h_{2}^{+}, \ldots, h_{r}^{+}\right)\right\} \neq 0$ in $H^{2}\left(\Gamma, \mathcal{G}_{+}\right)$we use an argument by contradiction. Suppose that there exists a cochain $h_{r+1}^{+}: \Gamma \rightarrow \mathcal{G}_{+}$such that

$$
-\partial h_{r+1}^{+}=\zeta_{r+1}\left(W+V_{+}, h_{2}^{+}, \ldots, h_{r}^{+}\right) .
$$

Then $h_{r+1}^{+}$factors through $N / N_{2}$ and defines a $\mathbb{C}$-linear map $\varphi_{r+1}^{+}$such that for all $y \in N / N_{2} \otimes \mathbb{C}$

$\left(\widetilde{E}_{r+1}\right)$

$$
\begin{array}{r}
\varphi_{r+1}^{+}\left(\left(t^{k}-\alpha^{k}\right) y\right)=-\zeta_{r+1}\left(W+V_{+}, \varphi_{2}^{+}, \ldots, \varphi_{r}^{+}\right)\left(\left(t^{k} y, 1\right),\left(0, t^{k}\right)\right) \\
+\zeta_{r+1}\left(W+V_{+}, \varphi_{2}^{+}, \ldots, \varphi_{r}^{+}\right)\left(\left(0, t^{k}\right),(y, 1)\right)
\end{array}
$$


On the other hand,

$$
\left\{\begin{aligned}
\left(t^{r}-\alpha^{r}\right) e_{r-2} & =r \alpha^{r-1} e_{r-1} \\
\left(t^{r-1}-\alpha^{r-1}\right) e_{r-2} & =(r-1) \alpha^{r-2} e_{r-1}
\end{aligned}\right.
$$

So, for $k=r$ and $y=e_{r-2}$, Equation $\left(\widetilde{E}_{r+1}\right)$ implies that

$$
\begin{aligned}
\varphi_{r+1}^{+}\left(r \alpha^{r-1} e_{r-1}\right)= & -\zeta_{r+1}\left(W+V_{+}, \varphi_{2}^{+}, \ldots, \varphi_{r}^{+}\right)\left(\left(\alpha^{r} e_{r-2}+r \alpha^{r-1} e_{r-1}, 1\right),\left(0, t^{r}\right)\right) \\
& +\zeta_{r+1}\left(W+V_{+}, \varphi_{2}^{+}, \ldots, \varphi_{r}^{+}\right)\left(\left(0, t^{r}\right),\left(e_{r-2}, 1\right)\right) \\
= & 2 r \alpha^{r} W(\mu) \varphi_{r}^{+}\left(e_{r-2}\right)+r^{2} \alpha^{r-1} W(\mu) \varphi_{r}^{+}\left(e_{r-1}\right) .
\end{aligned}
$$

For $k=r-1$ and $y=e_{r-2}$, Equation $\left(\widetilde{E}_{r+1}\right)$ implies

$$
\begin{aligned}
& \varphi_{r+1}^{+}\left((r-1) \alpha^{r-2} e_{r-1}\right) \\
& \quad=2(r-1) \alpha^{r-1} W(\mu) \varphi_{r}^{+}\left(e_{r-2}\right)+(r-1)^{2} W(\mu) \alpha^{r-2} \varphi_{r}^{+}\left(e_{r-1}\right) .
\end{aligned}
$$

So we obtain the system

$$
\left\{\begin{aligned}
r \alpha^{r-1} \varphi_{r+1}^{+}\left(e_{r-1}\right) & =2 r \alpha^{r} \varphi_{r}^{+}\left(e_{r-2}\right)+r^{2} \alpha^{r-1} \varphi_{r}^{+}\left(e_{r-1}\right) \\
(r-1) \alpha^{r-2} \varphi_{r+1}^{+}\left(e_{r-1}\right) & =2(r-1) \alpha^{r-1} \varphi_{r}^{+}\left(e_{r-2}\right)+(r-1)^{2} \alpha^{r-2} \varphi_{r}^{+}\left(e_{r-1}\right),
\end{aligned}\right.
$$

which is equivalent to

$$
\left\{\begin{aligned}
\varphi_{r+1}^{+}\left(e_{r-1}\right) & =2 \alpha \varphi_{r}^{+}\left(e_{r-2}\right)+r \varphi_{r}^{+}\left(e_{r-1}\right) \\
& =2 \alpha \varphi_{r}^{+}\left(e_{r-2}\right)+(r-1) \varphi_{r}^{+}\left(e_{r-1}\right) .
\end{aligned}\right.
$$

So $\varphi_{r}^{+}\left(e_{r-1}\right)=0=(2 \alpha)^{r-1} V_{+}\left(e_{0}\right)$ and $V_{+}\left(e_{0}\right)=0$ ie $V_{+}=0$ which is absurd. We conclude that $\left\{\zeta_{r+1}\left(W+V_{+}, h_{2}^{+}, \ldots, h_{r}^{+}\right)\right\} \neq 0$ in $H^{2}\left(\Gamma, \mathcal{G}_{+}\right)$.

\section{References}

[1] M Artin, On the solutions of analytic equations, Invent. Math. 5 (1968) 277-291 MR0232018

[2] L Ben Abdelghani, Arcs de représentations du groupe d'un noeud dans un groupe de Lie, PhD thesis, Université de Bourgogne (1998)

[3] L Ben Abdelghani, Espace des représentations du groupe d'un nœud classique dans un groupe de Lie, Ann. Inst. Fourier (Grenoble) 50 (2000) 1297-1321 MR1799747

[4] L Ben Abdelghani, D Lines, Involutions on knot groups and varieties of representations in a Lie group, J. Knot Theory Ramifications 11 (2002) 81-104 MR1885749 
[5] R C Blanchfield, Intersection theory of manifolds with operators with applications to knot theory, Ann. of Math. (2) 65 (1957) 340-356 MR0085512

[6] N Bourbaki, Éléments de mathématique. Fasc. XXXVII. Groupes et algèbres de Lie. Chapitre II: Algèbres de Lie libres. Chapitre III: Groupes de Lie, Actualités Scientifiques et Industrielles 1349, Hermann, Paris (1972) MR0573068

[7] S Boyer, On the local structure of $\mathrm{SL}(2, \mathbb{C})$-character varieties at reducible characters, Topology Appl. 121 (2002) 383-413 MR1909000

[8] KS Brown, Cohomology of groups, Graduate Texts in Math. 87, Springer, New York (1982) MR672956

[9] G Burde, Darstellungen von Knotengruppen, Math. Ann. 173 (1967) 24-33 MR0212787

[10] G Burde, H Zieschang, Knots, second edition, de Gruyter Studies in Math. 5, de Gruyter, Berlin (2003) MR1959408

[11] M Culler, P B Shalen, Varieties of group representations and splittings of 3-manifolds, Ann. of Math. (2) 117 (1983) 109-146 MR683804

[12] CD Frohman, EP Klassen, Deforming representations of knot groups in SU(2), Comment. Math. Helv. 66 (1991) 340-361 MR1120651

[13] C M Gordon, J Luecke, Knots are determined by their complements, J. Amer. Math. Soc. 2 (1989) 371-415 MR965210

[14] C M Herald, Existence of irreducible representations for knot complements with nonconstant equivariant signature, Math. Ann. 309 (1997) 21-35 MR1467643

[15] M Heusener, J Kroll, Deforming abelian SU(2)-representations of knot groups, Comment. Math. Helv. 73 (1998) 480-498 MR1633375

[16] M Heusener, J Porti, Deformations of reducible representations of 3-manifold groups into $\mathrm{PSL}_{2}(\mathbb{C})$, Algebr. Geom. Topol. 5 (2005) 965-997 MR2171800

[17] M Heusener, J Porti, E Suárez Peiró, Deformations of reducible representations of 3-manifold groups into $\mathrm{SL}_{2}$ (C), J. Reine Angew. Math. 530 (2001) 191-227 MR1807271

[18] E P Klassen, Representations of knot groups in SU(2), Trans. Amer. Math. Soc. 326 (1991) 795-828 MR1008696

[19] A Lubotzky, A R Magid, Varieties of representations of finitely generated groups, Mem. Amer. Math. Soc. 58 (1985) xi+117 MR818915

[20] A Nijenhuis, R W Richardson, Jr, Deformations of homomorphisms of Lie groups and Lie algebras, Bull. Amer. Math. Soc. 73 (1967) 175-179 MR0204575

[21] G de Rham, Introduction aux polynômes d'un næud, Enseignement Math. (2) 13 (1967) 187-194 (1968) MR0240804 
[22] D J Shors, Deforming reducible representations of knot groups in $\mathrm{SL}_{2}(\mathbb{C}), \mathrm{PhD}$ thesis, University of California Los Angeles (1991)

[23] A Weil, Remarks on the cohomology of groups, Ann. of Math. (2) 80 (1964) 149-157 MR0169956

[24] W Whitten, Knot complements and groups, Topology 26 (1987) 41-44 MR880506

Département de Mathématiques, Faculté des Sciences de Monastir Route de l'Environnement, 5000 Monastir, Tunisie

leila.benabdelghani@fsm.rnu.tn

Received: 24 March 2009 Revised: 6 November 2009 